\title{
Asymptotic behaviour of neuron population models structured by elapsed-time
}

\author{
José A. Cañizo Havva Yoldaş
}

November 8, 2018

\begin{abstract}
We study two population models describing the dynamics of interacting neurons, initially proposed by Pakdaman, Perthame, and Salort $(2010,2014)$. In the first model, the structuring variable $s$ represents the time elapsed since its last discharge, while in the second one neurons exhibit a fatigue property and the structuring variable is a generic "state". We prove existence of solutions and steady states in the space of finite, nonnegative measures. Furthermore, we show that solutions converge to the equilibrium exponentially in time in the case of weak nonlinearity (i.e., weak connectivity). The main innovation is the use of Doeblin's theorem from probability in order to show the existence of a spectral gap property in the linear (no-connectivity) setting. Relaxation to the steady state for the nonlinear models is then proved by a constructive perturbation argument.
\end{abstract}

\section{Contents}

1 Introduction 2

2 Spectral gaps in total variation $\quad 6$

3 An age-structured neuron population model $\quad 8$

3.1 Well posedness . . . . . . . . . . . . . . . . . . . . . 9 9

3.2 The linear equation . . . . . . . . . . . . . . . . . . . . . 14

3.2.1 Well-posedness ..................... . . 14

3.2.2 Positive lower bound . . . . . . . . . . . . . . . . . . . . 16

3.2 .3 Spectral gap . . . . . . . . . . . . . . . . 17

3.3 Stationary solutions for the nonlinear equation . . . . . . . . . . . . 17

3.4 Asymptotic behaviour . . . . . . . . . . . . . . . . 18

4 A structured neuron population model with fatigue 20

4.1 Well-posedness . . . . . . . . . . . . . . . 20

4.2 The linear equation . . . . . . . . . . . . . . . . . . 23

4.2.1 Well-posedness . . . . . . . . . . . . . . . 23

4.2.2 Positive lower bound . . . . . . . . . . . . . . . . 25

4.2 .3 Spectral gap . . . . . . . . . . . . . . . . . 26

4.3 Stationary solutions for the nonlinear equation . . . . . . . . . . . 27

4.4 Asymptotic behaviour . . . . . . . . . . . . . . . . . . . 28 


\section{Introduction}

Several mean-field models have been proposed to understand the electrical activity of a group of interacting neurons. They are all based on simplified models for the electrical activity of a single neuron, which give rise to an averaged partial differential equation (PDE) or integro-differential equation suitable when the number of neurons involved is large. In a rough approximation, neurons are assumed to undergo some sort of natural "charging" process, with a sudden "discharge" taking place in a stochastic way depending on the current charge, the time since the last discharge, and the activity of other connected neurons. A family of these models is structured by the membrane potential of neurons; that is, the quantity under study is the density $n(t, v)$ of neurons with potential $v$ at time $t$. The mathematical theory of these models is recent; see Tuckwell (1988); Brunel and Hakim (1999); Brunel (2000); Brette and Gerstner (2005); Rossant et al. (2011); Cáceres et al. (2011); Carrillo et al. (2013); Cáceres and Schneider (2017, 2018). The family of models we study in this paper is structured by the elapsed time since the last discharge and has been proposed in Pakdaman et al. (2010, 2013, 2014). The first model is based on stochastic simulations done in Pham et al. (1998). It is a nonlinear version of the conservative renewal equation (sometimes called as McKendrick-Von Foerster equation) which has been well-studied by many authors in the past as a model for a broad range of biological phenomena like epidemic spread and cell division (Perthame, 2006; Gyllenberg and Webb, 1990; Diekmann and Gyllenberg, 2008; Iannelli, 1995; Metz and Diekmann, 1986; Webb, 1985; Thieme, 2003). The second model has many similarities with a class of partial differential equations called growth-fragmentation equations. The nonlinear version we study here was introduced in Pakdaman et al. (2014), but on this general type of equations we also mention the works in Doumic-Jauffret and Gabriel (2010); Michel (2006); Perthame and Ryzhik (2005); Calvez et al. (2010); Engler et al. (2006); Farkas and Hagen (2007); Gabriel (2012); Laurençot and Walker (2007); Simonett and Walker (2006).

The dynamics of an age-structured, interacting neuron population is given by the integro-differential PDE

$$
\left\{\begin{array}{l}
\frac{\partial}{\partial t} n(t, s)+\frac{\partial}{\partial s} n(t, s)+p(N(t), s) n(t, s)=0, \quad t, s>0 \\
N(t):=n(t, s=0)=\int_{0}^{+\infty} p(N(t), s) n(t, s) d s, \quad t>0 \\
n(t=0, s)=n_{0}(s), \quad s \geq 0 .
\end{array}\right.
$$

which models the evolution of a neuron population density $n(t, s)$ depending on time $t$ and the time $s$ elapsed since the last discharge. Neurons randomly fire at a rate $p$ per unit of time, and they re-enter the cycle from $s=0$ immediately after they fire, as imposed through the boundary condition at $s=0$; the variable $s$ can thus be regarded as the 'age' of neurons, making a parallel with models for birth and death processes. The global activity $N(t)$ denotes the density of neurons which are undergoing a discharge at time $t$. If the firing rate $p$ increases with $N$, interactions are excitatory: the firing of neurons makes it more likely that connected neurons will also fire; if $p$ decreases with $N$ then interactions are inhibitory. This nonlinear model preserves positivity (see Section 3 for a rigorous proof) and has the conservation property

$$
\frac{d}{d t} \int_{0}^{+\infty} n(t, s) \mathrm{d} s=0 .
$$


In particular, this ensures that if the density of neurons is a probability distribution initially, then it remains so. Whenever it is convenient we assume that $n_{0}$ is a probability distribution (which may be assumed after a suitable scaling).

The second model we consider is a modified version of the first one where $s$ represents a generic "state" of the neuron, not necessarily the time elapsed since the last discharge. This model was proposed in Pakdaman et al. (2014), and assumes that neurons in a state $u$ return to a certain state $s<u$ after firing, with a certain probability distribution $\kappa(s, u)$. The model reads as follows:

$$
\left\{\begin{aligned}
\frac{\partial}{\partial t} n(t, s)+\frac{\partial}{\partial s} n(t, s) & +p(N(t), s) n(t, s) \\
= & \int_{0}^{+\infty} \kappa(s, u) p(N(t), u) n(t, u) d u, \quad u, s, t>0 \\
n(t, s=0) & =0, \quad N(t):=\int_{0}^{+\infty} p(N(t), s) n(t, s) d s, \quad t>0 \\
n(t=0, s) & =n_{0}(s), \quad s \geq 0 .
\end{aligned}\right.
$$

This equation differs from the first one in the addition of a kernel $\kappa=\kappa(s, u)$. For fixed $u$, the quantity $\kappa(\cdot, u)$ is a probability measure which gives the distribution of neurons which take the state $s$ when they discharge at a state $u$. Hence, neurons do not necessarily start the cycle from $s=0$ after firing, and so $s$ cannot be properly understood as an 'age' variable in this model. One should notice that equation (1) is a limiting case of equation $(3)$ when $\kappa(\cdot, u)=\delta_{0}(s)$, the Dirac delta at $s=0$. This connection is seen more obviously when a definition for weak or measure solutions is given; see Section 4. We remark that the terms involving $p$ and $\kappa$ are mathematically close to the ones appearing in fragmentation processes.

These equations and similar models have been shown to exhibit many interesting phenomena which are consistent with the experimental behaviour of neurons: depending on the parameter $p$ and the initial data one can find periodic solutions, apparently chaotic solutions, and solutions which approach an equilibrium state. The first two kinds of behaviour (periodic and chaotic solutions) are harder to study mathematically; numerical simulations have been performed in Pakdaman et al. (2010, 2013) and some explicit solutions have been found. Regarding convergence to equilibrium, some regimes are studied in these works using perturbative techniques, such as the so-called lowconnectivity and high-connectivity cases. Our contribution in this work is a simplified study of the low-connectivity case (corresponding to a weak nonlinearity) which gives improved results, and which uses a promising technique for this and similar models.

As a population balance equation of a form that appears often in mathematical biology, several techniques exist to study equations (1) and (3) rigorously. We refer to Perthame (2006) for a good exposition of many of the relevant tools. One of the main methods used so far in the study of convergence to equilibrium for equations (1) and (3) is the entropy method, which roughly consists in finding a suitable Lyapunov functional $H=H(n)$ such that

$$
\frac{\mathrm{d}}{\mathrm{d} t} H(n(t, \cdot))=-D(n(t, \cdot)) \leq 0
$$

along solutions $n=n(t, s)$ to (1) or (3), and then investigating whether one may prove inequalities of the type $\lambda H(n) \leq D(n)$ for some $\lambda>0$ and a family of functions $n$ sufficiently large to contain $n(t, \cdot)$ for all times $t$. If the answer is positive, one can apply 
the Gronwall inequality to $(4)$ and deduce that $H(n(t, \cdot))$ decays exponentially with a rate proportional to $e^{-\lambda t}$. This in turn may give useful information on the approach to equilibrium, often implying that $n(t, \cdot)$ approaches equilibrium in the $L^{1}$ norm. A fundamental difficulty is that phenomenological equations motivated by biological considerations do not have any obvious Lyapunov functionals. This difficulty leads us to considering cases which are close to a linear regime, taking advantage of the fact that mass- and positivity-conserving linear equations (essentially Markov evolutions) have a well-known family of Lyapunov functionals. This idea was followed in Pakdaman et al. (2010, 2013, 2014), using a specific Lyapunov functional obtained by integrating the primitive of $n-n_{*}$ (where $n_{*}$ is an equilibrium state) against a suitable weight.

Apart from the entropy method, for the time elapsed neuron network model (1), another approach has been developed in Mischler and Weng (2018); Weng (2015). This approach is based on spectral analysis theory for semigroups in Banach spaces. In Mischler and Weng (2018), uniqueness of the steady state and its nonlinear exponential stability in the weak connectivity regime for the first model was proved. This approach is extended in Weng (2015) to the cases without delay and with delay both in the weak and strong connectivity regimes considering a particular step function as a firing rate. Furthermore, in Chevallier et al. (2015) the link between several point processes models (Poisson, Wold, Hawkes) that have been proved to statistically fit real spike trains data and age-structured partial differential equations as introduced by Pakdaman et al. (2010) was investigated. This approach is extended to generalized Hawkes processes as microscopic models of individual neurons in Chevallier (2017).

We propose an alternative approach that is based on neither the entropy method nor the forementioned approaches, but instead takes advantage of a set of results in the theory of Markov processes known as Doeblin's theory, with some extensions such as Harris' theorem; see Harris (1956), or Hairer and Mattingly (2011); Gabriel (2018) for simplified recent proofs and Stroock (2005, Chapter 2) for a basic exposition. The idea is still based on first studying the linear case and then carrying out a perturbation argument; the difference is that we study the spectral properties of the linear operator by Doeblin's theory, which is quite flexible and later simplifies the proofs. We obtain a spectral gap property of the linear equation in a set of measures, and this leads to a perturbation argument which naturally takes care of the boundary conditions in (1)(3). Similar ideas are reviewed in Gabriel (2018) for the renewal equation, and have been recently used in Bansaye et al. (2017) for neuron population models structured by voltage.

Due to this strategy, studying solutions to (1) and (3) in the sense of measures comes as a natural setting for two important reasons: first, it fits well with the linear theory; and second, it allows us to treat the weakly nonlinear case as a perturbation of the linear one. Note that one difference between the weakly nonlinear case and the linear case for equation (1) is in the boundary condition, and this is conveniently encoded as a difference in a measure source term; see the proof of Theorem 1.1 for details on this. Measure solutions are also natural since a Delta function represents an initial population whose age (or structuring variable) is known precisely. There exist also recent works on numerical schemes for structured population models in the space of nonnegative measures (Carrillo et al., 2014; Brännström et al., 2013; Gwiazda et al., 2014). Entropy methods have also been extended to measure initial data by Gwiazda and Wiedemann (2016) for the renewal equation. 
Let us detail our notation and assumptions before stating the main results. Regarding the regularity of the firing rate $p=p(N, s)$, we always assume that $p$ is bounded, Lipschitz and nonnegative:

$$
p \in W^{1, \infty}([0,+\infty) \times[0,+\infty)), \quad p(N, s) \geq 0 \quad \text { for all } N, s \in[0,+\infty) .
$$

and we call $L$ the Lipschitz constant of $p$ with respect to $N$; that is, $L$ is the smallest number such that

$$
\left|p\left(N_{1}, s\right)-p\left(N_{2}, s\right)\right| \leq L\left|N_{1}-N_{2}\right| \text { for all } N_{1}, N_{2}, s \geq 0 .
$$

We assume that for a fixed global activity $N$ the firing rate increases as time passes; more precisely,

$$
\frac{\partial}{\partial s} p(N, s)>0, \quad \text { for all } N, s \geq 0 .
$$

where the derivative is defined. We also assume certain bounds on $p$ : there exist $s_{*}, p_{\min }, p_{\max }>0$ such that

$$
p_{\min } \mathbb{1}_{\left[s_{*}, \infty\right)} \leq p(N, s) \leq p_{\max } \text { for all } N, s \geq 0,
$$

where $\mathbb{1}_{A}$ denotes the characteristic function of a set $A$. Regarding $\kappa$, the basic modelling assumption is that

$$
\text { for each } u \geq 0, \kappa(\cdot, u) \text { is a probability measure supported on }[0, u] \text {. }
$$

We also impose a positivity condition on $\kappa$ which essentially states that after firing there is always a sizeable probability of jumping to a state with $s$ close to 0: there exist $\epsilon, 0<\delta<s_{*}$ such that

$$
\kappa(\cdot, u) \geq \epsilon \mathbb{1}_{[0, \delta]} \quad \text { for all } u \geq s_{*} .
$$

Our main result can be formulated as follows for both equation (1) and (3):

Theorem 1.1. We assume that (5)-(8) are satisfied for equation (1), or (5)-(10) for equation (3). We assume also that $L$ is small enough depending on $p$ and $\kappa$ (with an explicit estimate; see remarks after the statement).

Let $n_{0}$ be a probability measure on $[0,+\infty)$. There is a unique probability measure $n_{*}$ which is a stationary solution to (1) or (3), and there exist constants $C \geq 1, \lambda>0$ depending only on $p$ and $\kappa$ such that the (mild or weak) measure solution $n=n(t)$ to (1)-(3) satisfies

$$
\left\|n(t)-n_{*}\right\|_{\mathrm{TV}} \leq C e^{-\lambda t}\left\|n_{0}-n_{*}\right\|_{\mathrm{TV}}, \text { for all } t \geq 0 .
$$

Remark 1.2. The constants are all constructive. To be precise, one can take

$$
\begin{array}{lll}
\lambda=\lambda_{1}-\tilde{C}, & C=C_{1} & \text { for (1), } \\
\lambda=\lambda_{2}-\tilde{C}, & C=C_{2} & \text { for (3), }
\end{array}
$$

where

$$
\begin{aligned}
C_{1} & :=\frac{1}{1-s_{*} \beta}, & \lambda_{1} & =-\frac{\log \left(1-s_{*} \beta\right)}{2 s_{*}} \\
C_{2} & :=\frac{1}{1-\epsilon \delta\left(s_{*}-\delta\right) \beta}, & \lambda_{2} & =-\frac{\log \left(1-\epsilon \delta\left(s_{*}-\delta\right) \beta\right)}{2 s_{*}}
\end{aligned}
$$


and with

$$
\beta=p_{\min } e^{-2 p_{\max } s_{*}} \text { and } \tilde{C}=2 p_{\max } \frac{L}{1-L} .
$$

The smallness condition on $L$ can be written as

$$
L<\min \left\{\frac{p_{\min }^{2}}{p_{\max }^{2}\left(s_{*} p_{\min }\left(s_{*} p_{\min }+2\right)+2\right)}, \frac{\log \left(1-s_{*} \beta\right)}{\log \left(1-s_{*} \beta\right)-4 p_{\max } s_{*}}\right\} \text { for }(1)
$$

or

$$
L<\min \left\{\frac{p_{\min } \epsilon \delta\left(s_{*}-\delta\right) \beta}{p_{\min } \epsilon \delta\left(s_{*}-\delta\right) \beta+p_{\max } e^{4 p_{\max } s_{*}}}, \frac{\log \left(1-\epsilon \delta\left(s_{*}-\delta\right)\right)}{\log \left(1-\epsilon \delta\left(s_{*}-\delta\right)\right)-4 p_{\max } s_{*}}\right\} \text { for (3). }
$$

As remarked above, the closest results in the literature are those of Pakdaman et al. (2010, 2014). Our equation (1) is essentially the model in Pakdaman et al. (2010), written in a slightly different formulation that does not include time delay and does not highlight the connectivity as a separate parameter (the connectivity of neurons in our case is measured in the size of $\partial_{N} p$ ). The results in Pakdaman et al. (2010) use entropy methods and show exponential convergence to equilibrium (a similar statement to Theorem 1.1) in a weighted $L^{1}$ space, for the case with delay and for a particular form of the firing rate $p$. As compared to this, our results work in a space of measures and can be easily written for general firing rates $p$; however, we have not considered the large-connectivity case (which would correspond to large $\partial_{N} p$ in our case) or the effects of time delay.

Similar remarks apply to the results for equation (3) contained in Pakdaman et al. (2014). In this case our strategy gives in general conditions which are simpler to state, and provide a general framework which may be applied to similar models. Again, we have not considered a time delay in the equation, which is a difference with the above work. There are numerical simulations and further results on regimes with a stronger nonlinearity in Pakdaman et al. (2010, 2013, 2014).

This paper is organized as follows. In Section 2 we state Doeblin's Theorem from the theory of Markov processes, which plays a crucial role in our convergence results. Section 3 and section 4 are dedicated to the age-structured neuron population model (1) and the structured neuron population model with a fatigue (3) respectively. The organisation of those last two sections is the same: in each, we first give proofs for wellposedness, and existence and uniqueness of stationary solutions, always in the weak nonlinearity regime. Later we consider the linear equations (when $p$ does not depend on $N$ ) and we prove that solutions have positive lower bounds which ensures that the associated stochastic semigroups satisfy the Doeblin condition, obtaining exponential convergence results for the linear problems. Finally, we prove exponential relaxation to the steady state for the nonlinear models (1)-(3) by a perturbation argument based on the linear theory.

\section{Spectral gaps in total variation}

We briefly present here the version of Doeblin's theorem that we use in this paper. A recent proof which brought it to our attention can be found in ?; a general version known as Harris' theorem can be found in Harris (1956); Hairer and Mattingly (2011). 
This result applies to stochastic semigroups defined in a space of measures (or in an $L^{1}$ space); that is, mass- and positivity-preserving semigroups ${ }^{1}$.

Given a measurable space $(E, \mathcal{A})$ we denote by $\mathcal{M}(E)$ the set of finite measures on $E$, and by $\mathcal{P}(E)$ the set of probability measures on $E$. We equip $\mathcal{M}(E)$ with the usual total variation norm which we denote by $\|\cdot\|_{\mathrm{TV}}$; we recall that this norm is defined by $\|\mu\|:=\int_{E} \mu_{+}+\int_{E} \mu_{-}$, where $\mu=\mu_{+}-\mu_{-}$is the Hahn-Jordan decomposition of the measure $\mu$ into its positive and negative parts.

Definition 2.1. Let $(E, \mathcal{A})$ be a measurable space. A stochastic operator on $\mathcal{M}(E)$ is a linear operator $S: \mathcal{M}(E) \rightarrow \mathcal{M}(E)$ such that $S \mu \geq 0$ for all $\mu \geq 0$, and such that $\int_{E} S \mu=\int_{E} \mu$ for all $\mu \in \mathcal{M}(E)$. Equivalently, it is a linear operator which preserves the space $\mathcal{P}(E)$ of probability measures on $E$.

A stochastic semigroup on $\mathcal{M}(E)$ is a semigroup $\left(S_{t}\right)_{t \geq 0}$ of operators $S_{t}: \mathcal{M}(E) \rightarrow$ $\mathcal{M}(E)$ such that $S_{t}$ is a stochastic operator for each $t \geq 0$. A stationary state of a semigroup $\left(S_{t}\right)_{t \geq 0}$ on $\mathcal{M}(E)$ is a measure $\mu \in \mathcal{M}(E)$ such that $S_{t} \mu=\mu$ for all $t \geq 0$.

We observe that $\left\|S_{t} \mu\right\|_{\mathrm{TV}} \leq\|\mu\|_{\mathrm{TV}}$ holds true for all $\mu \in \mathcal{M}(E)$. Since $S_{t}$ preserves order, we have $-S_{t}|\mu| \leq S_{t} \mu \leq S_{t}|\mu|$ which implies $\left|S_{t} \mu\right| \leq S_{t}|\mu|$. Then we integrate both sides to obtain $\int\left|S_{t} \mu\right| \leq \int S_{t} \mu=\int|\mu|$ for all $\mu \in \mathcal{M}(E)$ by using the mass conservation property of $S_{t}$.

Definition 2.2. We say a stochastic operator $S$ satisfies the Doeblin condition when there exist $0<\alpha<1$ and a probability measure $\nu$ on $(E, \mathcal{A})$ such that

$$
S \mu \geq \alpha \nu \quad \text { for all } \mu \in \mathcal{P}(E) \text {. }
$$

The following version of Doeblin's theorem is essentially the same as the one in Gabriel (2018) or Stroock (2005, Chapter 2), and a particular case of Hairer and Mattingly (2011) if one ignores some technical conditions regarding the existence of a kernel for the semigroup. We give a short proof here for clarity, essentially along the lines of Gabriel (2018).

Theorem 2.3 (Semigroup version of Doeblin's theorem). Let $(E, \mathcal{A})$ be a measurable space and $\left(S_{t}\right)_{t \geq 0}$ a stochastic semigroup on $\mathcal{M}(E)$. If there exists $t_{0}>0$ such that $S_{t_{0}}$ satisfies the Doeblin condition (12) then the semigroup has a unique equilibrium $n_{*}$ in $\mathcal{P}(E)$, and

$$
\left\|S_{t}\left(n-n_{*}\right)\right\|_{\mathrm{TV}} \leq \frac{1}{1-\alpha} e^{-\lambda t}\left\|n-n_{*}\right\|_{\mathrm{TV}}, \text { for all } t \geq 0,
$$

for all $n \in \mathcal{P}(E)$, where

$$
\lambda:=-\frac{\log (1-\alpha)}{t_{0}}>0
$$

In addition,

$$
\left\|S_{t_{0}}\left(n_{1}-n_{2}\right)\right\|_{\mathrm{TV}} \leq(1-\alpha)\left\|n_{1}-n_{2}\right\|_{\mathrm{TV}}
$$

for any probability measures $n_{1}, n_{2}$ on $E$.

\footnotetext{
${ }^{1}$ In the literature a Markov semigroup is often the dual of this kind of semigroup, but is sometimes also the same as our definition of a stochastic semigroup. We give the definition to avoid any confusion.
} 
Proof. By the triangle inequality we have

$$
\left\|S_{t_{0}} \mu_{1}-S_{t_{0}} \mu_{2}\right\|_{\mathrm{TV}} \leq\left\|S_{t_{0}} \mu_{1}-\alpha \nu\right\|_{\mathrm{TV}}+\left\|S_{t_{0}} \mu_{2}-\alpha \nu\right\|_{\mathrm{TV}}
$$

Now, since $S_{t_{0}} \mu_{1} \geq \alpha \nu$, we can write

$$
\left\|S_{t_{0}} \mu_{1}-\alpha \nu\right\|_{\mathrm{TV}}=\int\left(S_{t_{0}} \mu_{1}-\alpha \nu\right)=\int \mu_{1}-\alpha=1-\alpha,
$$

due to mass conservation, and similarly for the term $\left\|S_{t_{0}} \mu_{2}-\alpha \nu\right\|_{\mathrm{TV}}$. This gives

$$
\left\|S_{t_{0}} \mu_{1}-S_{t_{0}} \mu_{2}\right\|_{\mathrm{TV}} \leq 2(1-\alpha)=(1-\alpha)\left\|\mu_{1}-\mu_{2}\right\|_{\mathrm{TV}}
$$

if $\mu_{1}, \mu_{2} \in \mathcal{P}(E)$ have disjoint support. By homogeneity, this inequality is obviously also true for any nonnegative $\mu_{1}, \mu_{2} \in \mathcal{M}(E)$ having disjoint support with $\int \mu_{1}=\int \mu_{2}$. We obtain the inequality in general for any $\mu_{1}, \mu_{2} \in \mathcal{M}(E)$ with the same integral by writing $\mu_{1}-\mu_{2}=\left(\mu_{1}-\mu_{2}\right)_{+}-\left(\mu_{2}-\mu_{1}\right)_{+}$, which is a difference of nonnegative measures with the same integral. This shows (14).

The contractivity (15) shows that the operator $S_{t_{0}}$ has a unique fixed point in $\mathcal{P}(E)$, which we call $n_{*}$. In fact, $n_{*}$ is a stationary state of the whole semigroup since for all $s \geq 0$ we have

$$
S_{t_{0}} S_{s} n_{*}=S_{s} S_{t_{0}} n_{*}=S_{s} n_{*}
$$

which shows that $S_{s} n_{*}$ (which is again a probability measure) is also a stationary state of $S_{t_{0}}$; due to uniqueness,

$$
S_{s} n_{*}=n_{*} .
$$

Hence the only stationary state of $\left(S_{t}\right)_{t \geq 0}$ must be $n_{*}$, since any stationary state of $\left(S_{t}\right)_{t \geq 0}$ is in particular a stationary state of $S_{t_{0}}$.

In order to show (13), for any $n \in \mathcal{P}(E)$ and any $t \geq 0$ we write

$$
k:=\left\lfloor t / t_{0}\right\rfloor,
$$

(where $\lfloor\cdot\rfloor$ denotes the integer part) so that

$$
\frac{t}{t_{0}}-1<k \leq \frac{t}{t_{0}} .
$$

Then,

$$
\begin{aligned}
\left\|S_{t}\left(n-n_{*}\right)\right\|_{\mathrm{TV}} & =\left\|S_{t-k t_{0}} S_{k t_{0}}\left(n-n_{*}\right)\right\|_{\mathrm{TV}} \leq\left\|S_{k t_{0}}\left(n-n_{*}\right)\right\|_{\mathrm{TV}} \\
& \leq(1-\alpha)^{k}\left\|n-n_{*}\right\|_{\mathrm{TV}} \leq \frac{1}{1-\alpha} \exp \left(\frac{t \log (1-\alpha)}{t_{0}}\right)\left\|n-n_{*}\right\|_{\mathrm{TV}} .
\end{aligned}
$$

\section{An age-structured neuron population model}

In this section we consider equation (1) for an age-structured neuron population. We first develop a well-posedness theory in the sense of measures, and then we use Doeblin's Theorem 2.3 for the linear problem (32) to show exponential convergence to the equilibrium. After giving conditions for existence and uniqueness of a stationary solution to equation (1), we use a perturbation argument in order to obtain a result on its asymptotic behaviour. 


\subsection{Well posedness}

In order to develop our well-posedness theory in measures we need to introduce our notation and the norms we will be considering. We denote $\mathbb{R}_{0}^{+}:=[0,+\infty)$, and $\mathcal{M}\left(\mathbb{R}_{0}^{+}\right)$ is the set of finite, signed Borel measures on $\mathbb{R}_{0}^{+} \cdot \mathcal{M}_{+}\left(\mathbb{R}_{0}^{+}\right)$denoted the subset of $\mathcal{M}\left(\mathbb{R}_{0}^{+}\right)$ formed by the nonnegative measures. Since we will always work in $\mathbb{R}_{0}^{+}$, for simplicity we will often write $\mathcal{M}$ and $\mathcal{M}_{+}$to denote these sets, respectively.

We often identify a measure $\mu \in \mathcal{M}\left(\mathbb{R}_{0}^{+}\right)$with its density with respect to Lebesgue measure, denoting the latter by the function $\mu=\mu(s)$. We abuse notation by writing $\mu(s)$ even for measures that may not have a density with respect to Lebesgue measure. Similarly, for a function $n:[0, T) \rightarrow \mathcal{M}\left(\mathbb{R}_{0}^{+}\right)$we may often write $n(t, s)$ even if $n(t)$ does not have a density with respect to Lebesgue measure. In these cases any identities involved should be understood as identities between measures.

We denote by $\mathcal{C}_{0}\left(\mathbb{R}_{0}^{+}\right) \equiv \mathcal{C}_{0}$ the set of continuous functions $\phi$ on $\mathbb{R}_{0}^{+}$with $\lim _{s \rightarrow+\infty} \phi(s)=$ 0 endowed with the supremum norm

$$
\|\phi\|_{\infty}:=\sup _{s \geq 0}|\phi(s)|
$$

$\mathcal{C}_{0}\left(\mathbb{R}_{0}^{+}\right)$becomes a Banach space. Similarly, $\mathcal{C}_{\mathrm{c}}\left(\mathbb{R}_{0}^{+}\right) \equiv \mathcal{C}_{\mathrm{c}}$ denotes the set of compactly supported continuous functions on $[0,+\infty)$.

In $\mathcal{M}$ one can define the usual total variation norm, which we will denote by $\|\cdot\|_{\mathrm{TV}}$. We recall that $\left(\mathcal{M},\|\cdot\|_{\mathrm{TV}}\right)$ is a Banach space, and is the topological dual of $\mathcal{C}_{0}([0,+\infty))$ with the supremum norm, as stated by the Riesz representation theorem. The weak* topology on $\mathcal{M}$ is the weakest topology that makes all functionals $T: \mathcal{M} \rightarrow \mathbb{R}$, $\mu \mapsto \int_{\mathbb{R}_{0}^{+}} \phi \mu$ continuous, for all $\phi \in \mathcal{C}_{0}$. In the associated topology, a sequence $\left(\mu_{k}\right)_{k \geq 1}$ in $\mathcal{M}$ converges in the weak-* sense to $\mu \in \mathcal{M}$ when

$$
\lim _{k \rightarrow+\infty} \int_{\mathbb{R}_{0}^{+}} \phi \mu_{k}=\int_{\mathbb{R}_{0}^{+}} \phi \mu \quad \text { for all } \phi \in \mathcal{C}_{0} .
$$

We will also use the bounded Lipschitz norm $\|\cdot\|_{\mathrm{BL}}$ on $\mathcal{M}$, sometimes known as the flat metric or the $W^{1, \infty}$ dual metric, defined by

$$
\|\mu\|_{\mathrm{BL}}:=\sup _{\psi \in \mathcal{L}} \int_{\mathbb{R}_{0}^{+}} \psi \mu, \quad \mu \in \mathcal{M}
$$

where

$$
\mathcal{L}:=\left\{\psi \in \mathcal{C}\left(\mathbb{R}_{0}^{+}\right) \mid \psi \text { bounded and Lipschitz with }\|\psi\|_{\infty}+\left\|\psi^{\prime}\right\|_{\infty} \leq 1\right\} .
$$

One sees from this definition that the bounded Lipschitz norm is dual to the norm

$$
\|\psi\|_{1, \infty}:=\|\psi\|_{\infty}+\left\|\psi^{\prime}\right\|_{\infty}, \quad \psi \in W^{1, \infty}\left(\mathbb{R}_{0}^{+}\right)
$$

defined on $W^{1, \infty}\left(\mathbb{R}_{0}^{+}\right)=\left\{\psi \in \mathcal{C}\left(\mathbb{R}_{0}^{+}\right) \mid \psi\right.$ bounded and Lipschitz $\}$ (but $\left(\mathcal{M},\|\cdot\|_{\mathrm{BL}}\right)$ is not the topological dual of $\left.W^{1, \infty}\right)$. An important property of this norm is that it metrises the weak-* topology on any tight set with bounded total variation. We recall that a set $B \subseteq \mathcal{M}$ is tight if for every $\epsilon>0$ there exists $R>0$ such that $|\mu|((R,+\infty))<\epsilon$ for all $\mu \in B$. 
Lemma 3.1 (Lorenz (2010), 2.5.1, Proposition 43). If $B \subseteq \mathcal{M}$ is tight and is bounded in total variation norm, then the topology associated to $\|\cdot\|_{\mathrm{BL}}$ on $B$ is equal to the weak-* topology on B.

If $I \subseteq \mathbb{R}$ is an interval we denote by $\mathcal{C}\left(I, \mathcal{M}_{+}\left(\mathbb{R}_{0}^{+}\right)\right)$the set of functions $n: I \rightarrow$ $\mathcal{M}_{+}\left(\mathbb{R}_{0}^{+}\right)$which are continuous with respect to the bounded Lipschitz norm on $\mathcal{M}_{+}\left(\mathbb{R}_{0}^{+}\right)$. We define mild measure solutions to equation (1) by the usual procedure of rewriting it using Duhamel's formula. We denote by $\left(T_{t}\right)_{t \geq 0}$ the translation semigroup generated on $\left(\mathcal{M},\|\cdot\|_{\mathrm{BL}}\right)$ by the operator $-\partial_{s}$. That is: for $t \geq 0$, any measure $n \in \mathcal{M}\left(\mathbb{R}_{0}^{+}\right)$and any $\phi \in \mathcal{C}_{0}\left(\mathbb{R}_{0}^{+}\right)$,

$$
\int_{\mathbb{R}_{0}^{+}} \phi(s) T_{t} n(s) \mathrm{d} s:=\int_{\mathbb{R}_{0}^{+}} \phi(s+t) n(s) \mathrm{d} s .
$$

In other words, using the notation we follow in this paper,

$$
T_{t} n(s):=n(s-t)
$$

with the understanding that $n$ is zero on $(-\infty, 0)$.

Definition 3.2. Assume $p$ satisfies (5) and is nonnegative. A couple of functions $n \in \mathcal{C}\left([0, T), \mathcal{M}_{+}\left(\mathbb{R}_{0}^{+}\right)\right)$and $N \in \mathcal{C}([0, T),[0,+\infty))$, defined on an interval $[0, T)$ for some $T \in(0,+\infty)$, is called a mild measure solution to (1) with initial data $n_{0} \in \mathcal{M}\left(\mathbb{R}_{0}^{+}\right)$ and $N_{0} \in \mathbb{R}$ if it satisfies $n(0)=n_{0}, N(0)=N_{0}$,

$$
n(t, s)=T_{t} n_{0}(s)-\int_{0}^{t} T_{t-\tau}(p(N(\tau), \cdot) n(\tau, \cdot))(s) \mathrm{d} \tau+\int_{0}^{t} T_{t-\tau}\left(N(\tau) \delta_{0}\right)(s) \mathrm{d} \tau
$$

for all $t \in[0, T)$, and

$$
N(t)=\int_{0}^{\infty} p(N(t), s) n(t, s) \mathrm{d} s, \quad t \in[0, T) .
$$

Remark 3.3. We notice that the second term in (17) can be rewritten as

$$
\begin{aligned}
\int_{0}^{t} T_{t-\tau}\left(N(\tau) \delta_{0}\right)(s) \mathrm{d} \tau=\int_{0}^{t} N(\tau) \delta_{t-\tau}(s) \mathrm{d} \tau & \\
& =N(t-s) \mathbb{1}_{[0, t]}(s)=N(t-s) \mathbb{1}_{[0, \infty)}(t-s)
\end{aligned}
$$

This will sometimes be a more convenient form.

By integrating in $\mathbb{R}_{0}^{+}$, Definition 3.2 directly implies mass conservation:

Lemma 3.4 (Mass conservation for measure solutions). Let $T \in(0,+\infty]$. Any mild measure solution $(n, N)$ to $(1)$ defined on $[0, T)$ satisfies

$$
\int_{\mathbb{R}_{0}^{+}} n(t, s) \mathrm{d} s=\int_{\mathbb{R}_{0}^{+}} n_{0}(s) \mathrm{d} s, \quad \text { for all } t \in[0, T),
$$

or in other words (since solutions are nonnegative measures by definition),

$$
\|n(t)\|_{\mathrm{TV}}=\left\|n_{0}\right\|_{\mathrm{TV}} \quad \text { for all } t \in[0, T) .
$$


Lemma 3.5. Assume that $p$ satisfies (5) and the Lipschitz constant $L$ in (6) satisfies $L<1 /\|n\|_{\mathrm{TV}}$, and let $n \in \mathcal{M}\left(\mathbb{R}_{0}^{+}\right)$. There exists a unique $N \in \mathbb{R}$ satisfying

$$
N=\int_{0}^{\infty} p(N, s) n(s) \mathrm{d} s .
$$

Under these conditions, if $n_{1}, n_{2} \in \mathcal{M}\left(\mathbb{R}_{0}^{+}\right)$are two measures and $N_{1}, N_{2} \in \mathbb{R}$ are the corresponding solutions to (20), then

$$
\left|N_{1}-N_{2}\right| \leq \frac{\|p\|_{\infty}}{1-L\left\|n_{1}\right\|_{\mathrm{TV}}}\left\|n_{1}-n_{2}\right\|_{\mathrm{TV}}
$$

Proof. We define the map $\Phi: \mathbb{R} \rightarrow \mathbb{R}$ by

$$
\Phi(N):=\int_{0}^{\infty} p(N, s) n(s) \mathrm{d} s,
$$

and we notice that for any $N_{1}, N_{2} \in \mathbb{R}$,

$$
\left|\Phi\left(N_{1}\right)-\Phi\left(N_{2}\right)\right| \leq\left\|p\left(N_{1}, \cdot\right)-p\left(N_{2}, \cdot\right)\right\|_{\infty}\|n\|_{\mathrm{TV}} \leq L\left|N_{1}-N_{2}\right|\|n\|_{\mathrm{TV}} .
$$

Since $L<1 /\|n\|_{\mathrm{TV}}$, the map $\Phi$ is contractive and has a unique fixed point, which is a solution to (20). For the second part of the lemma, consider $n_{1}, n_{2} \in \mathcal{M}\left(\mathbb{R}_{0}^{+}\right)$and $N_{1}$, $N_{2}$ the corresponding solutions to (20). Then

$$
\begin{array}{r}
\left|N_{1}-N_{2}\right| \leq \int_{0}^{\infty}\left|p\left(N_{1}, s\right)-p\left(N_{2}, s\right)\right| n_{1}(s) \mathrm{d} s+\left|\int_{0}^{\infty} p\left(N_{2}, s\right)\left(n_{1}(s)-n_{2}(s)\right) \mathrm{d} s\right| \\
\leq L\left|N_{1}-N_{2}\right|\left\|n_{1}\right\|_{\mathrm{TV}}+\|p\|_{\infty}\left\|n_{1}-n_{2}\right\|_{\mathrm{TV}},
\end{array}
$$

which shows (21).

Theorem 3.6 (Well-posedness of (1) in measures). Assume that $p$ satisfies (5) and the Lipschitz constant $L$ in (6) satisfies $L \leq 1 /\left(4\left\|n_{0}\right\|_{\mathrm{TV}}\right)$. For any given initial data $n_{0} \in \mathcal{M}_{+}\left(\mathbb{R}_{0}^{+}\right)$there exists a unique measure solution $n \in \mathcal{C}\left([0,+\infty) ; \mathcal{M}_{+}\left(\mathbb{R}_{0}^{+}\right)\right)$of $(1)$ in the sense of Definition 3.2. In addition, if $n_{1}, n_{2}$ are any two mild measure solutions to (1) (with possibly different initial data) defined on any interval $[0, T)$ then

$$
\left\|n_{1}(t)-n_{2}(t)\right\|_{\mathrm{TV}} \leq\left\|n_{1}(0)-n_{2}(0)\right\|_{\mathrm{TV}} e^{4\|p\|_{\infty} t} \quad \text { for all } t \in[0, T) .
$$

Remark 3.7. We notice that the condition that $L$ is small is already needed here, since otherwise the problem is not well-posed: consider for example the case $p(N, s):=N$, for which a solution should satisfy

$$
N(t)=N(t) \int_{0}^{\infty} n(t, s) \mathrm{d} s
$$

which only allows two options: either $N(t)=0$ or $\int_{0}^{\infty} n(t, s) \mathrm{d} s=1$. If $\int_{0}^{\infty} n_{0}(s) \mathrm{d} s=1$ then the second option holds and there are infinitely many solutions (since the choice of $N=N(t)$ is free). If $\int_{0}^{\infty} n_{0}(s) \mathrm{d} s \neq 1$ then $N(t)$ must be 0 for all $t>0$. In this latter case, either $N_{0}=0$ (and then the only solution is just pure transport: $n(t, s)=n_{0}(s-t)$ for $s>t, n(t, s)=0$ otherwise) or $N_{0} \neq 0$ (and there there are no solutions).

Similar ill-posed examples can be easily designed with firing rates of the form $p(N, s)=f(N) g(s)$. 
Proof of Theorem 3.6. The proof of this result is a standard fixed-point argument as followed for example in Cañizo et al. (2013), or in Pakdaman et al. (2010) for $L^{1}$ solutions.

Let us first show existence of a solution for a nonnegative initial measure $n_{0} \in$ $\mathcal{M}_{+}\left(\mathbb{R}_{0}^{+}\right)$. If $n_{0}=0$ it is clear that setting $n(t)$ equal to the zero measure on $\mathbb{R}_{0}^{+}$for all $t$ defines a solution, so we assume $n_{0} \neq 0$. Fix $C, T>0$, to be chosen later. Consider the complete metric space

$$
\mathcal{X}=\left\{n \in \mathcal{C}\left([0, T], \mathcal{M}_{+}\left(\mathbb{R}_{0}^{+}\right)\right) \mid n(0)=n_{0},\|n(t)\|_{\mathrm{TV}} \leq C \text { for all } t \in[0, T]\right\},
$$

endowed with the norm

$$
\|n\|_{\mathcal{X}}:=\sup _{t \in[0, T]}\|n(t)\|_{\mathrm{TV}}
$$

We remark that $\mathcal{C}\left([0, T], \mathcal{M}\left(\mathbb{R}_{0}^{+}\right)\right)$refers to functions which are continuous in the bounded Lipschitz topology, not in the total variation one. Define an operator $\Psi: \mathcal{X} \rightarrow \mathcal{X}$ by

$$
\Psi[n](t):=T_{t} n_{0}-\int_{0}^{t} T_{t-\tau}(p(N(\tau), \cdot) n(\tau)) \mathrm{d} \tau+\int_{0}^{t} T_{t-\tau}\left(N(\tau) \delta_{0}\right) \mathrm{d} \tau
$$

for all $n \in \mathcal{X}$, where $N(t)$ is defined implicitly (see Lemma 3.5) as

$$
N(t)=\int_{0}^{\infty} p(N(t), s) n(t, s) \mathrm{d} s \quad \text { for } t \in[0, T] .
$$

The definition of $\Psi[n]$ indeed makes sense, since both $\tau \mapsto T_{t-\tau}(p(N(\tau), \cdot) n(\tau))$ and $\tau \mapsto T_{t-\tau}\left(N(\tau) \delta_{0}\right)$ are continuous functions from $[0, T]$ to $\mathcal{M}\left(\mathbb{R}_{0}^{+}\right)$, hence integrable (in the sense of the Bochner integral).

We first check that $\Psi[n]$ is indeed in $\mathcal{X}$. It is easy to see that $t \mapsto \Psi[n](t)$ is continuous in the bounded Lipschitz topology, and it is a nonnegative measure for each $t \in[0, T]$. We also have

$$
\begin{aligned}
\|\Psi[n](t)\|_{\mathrm{TV}} \leq\left\|n_{0}\right\|_{\mathrm{TV}} & +\int_{0}^{t}\|p(N(\tau), \cdot) n(\tau, \cdot)\|_{\mathrm{TV}} \mathrm{d} \tau+\int_{0}^{t}\left\|N(\tau) \delta_{0}\right\|_{\mathrm{TV}} \mathrm{d} \tau \\
& \leq\left\|n_{0}\right\|_{\mathrm{TV}}+T C\|p\|_{\infty}+T\|N\|_{L^{\infty}([0, T])} \leq\left\|n_{0}\right\|_{\mathrm{TV}}+2 T C\|p\|_{\infty}
\end{aligned}
$$

We choose

so that

$$
T \leq \frac{1}{4\|p\|_{\infty}} \quad \text { and } \quad C:=2\left\|n_{0}\right\|_{\mathrm{TV}}
$$

$$
\left\|n_{0}\right\|_{\mathrm{TV}}+2 T C\|p\|_{\infty} \leq\left\|n_{0}\right\|_{\mathrm{TV}}+\frac{C}{2} \leq C .
$$

Hence with these conditions on $T$ and $C$ we have $\Psi[n] \in \mathcal{X}$.

Let us show that $\Psi$ is a contraction mapping. Take $n_{1}, n_{2} \in \mathcal{X}$ and let $N_{1}, N_{2}$ be defined by (24) corresponding to $n_{1}$ and $n_{2}$, respectively. We have

$$
\begin{array}{r}
\left\|\Psi\left[n_{1}\right](t)-\Psi\left[n_{2}\right](t)\right\|_{\mathrm{TV}} \leq \int_{0}^{t}\left\|\left(p\left(N_{1}(\tau), \cdot\right)-p\left(N_{2}(\tau), \cdot\right)\right) n_{1}(\tau, \cdot)\right\|_{\mathrm{TV}} \mathrm{d} \tau \\
+\int_{0}^{t}\left\|p\left(N_{2}(\tau), \cdot\right)\left(n_{1}(\tau)-n_{2}(\tau)\right)\right\|_{\mathrm{TV}} \mathrm{d} \tau+\int_{0}^{t}\left\|\left(N_{1}(\tau)-N_{2}(\tau)\right) \delta_{0}\right\|_{\mathrm{TV}} \mathrm{d} \tau \\
=: T_{1}+T_{2}+T_{3} .
\end{array}
$$


We bound each term separately. For $T_{1}$, since $L \leq 1 /\left(4\left\|n_{0}\right\|_{\mathrm{TV}}\right)$, using Lemma 3.5 we have

$$
\begin{aligned}
T_{1} \leq T C L \sup _{\tau \in[0, T]}\left|N_{1}(\tau)-N_{2}(\tau)\right| & \\
& \leq 2 T C L\|p\|_{\infty}\left\|n_{1}-n_{2}\right\|_{\mathcal{X}} \leq T\|p\|_{\infty}\left\|n_{1}-n_{2}\right\|_{\mathcal{X}}
\end{aligned}
$$

For $T_{2}$,

$$
T_{2} \leq T\|p\|_{\infty}\left\|n_{1}-n_{2}\right\|_{\mathcal{X}}
$$

and for $T_{3}$, using again Lemma 3.5,

$$
T_{3} \leq T \sup _{\tau \in[0, T]}\left|N_{1}(\tau)-N_{2}(\tau)\right| \leq 2 T\|p\|_{\infty}\left\|n_{1}-n_{2}\right\|_{\mathcal{X}}
$$

Putting equations (26)-(28) together and taking the supremum over $0 \leq t \leq T$,

$$
\left\|\Psi\left[n_{1}\right]-\Psi\left[n_{2}\right]\right\|_{\mathcal{X}} \leq 4 T\|p\|_{\infty}\left\|n_{1}-n_{2}\right\|_{\mathcal{X}} .
$$

Taking now $T \leq 1 /\left(8\|p\|_{\infty}\right)$ ensures that $\Psi$ is contractive, so it has a unique fixed point in $\mathcal{X}$, which is a mild measure solution on $[0, T]$. If we call $n$ this fixed point, since $\|n(T)\|_{\mathrm{TV}}=\left\|n_{0}\right\|_{\mathrm{TV}}$ by mass conservation (see Lemma 3.4), we may repeat this argument to continue the solution on $[T, 2 T],[2 T, 3 T]$, showing that there is a solution defined on $[0,+\infty)$.

In order to show stability of solutions with respect to the initial data (which implies uniqueness of solutions), take two measures $n_{0}^{1}, n_{0}^{2} \in \mathcal{M}_{+}\left(\mathbb{R}_{0}^{+}\right)$, and consider two solutions $n_{1}, n_{2}$ with initial data $n_{0}^{1}, n_{0}^{2}$ respectively. We have

$$
\begin{aligned}
\| n_{1}(t) & -n_{2}(t)\left\|_{\mathrm{TV}} \leq\right\| n_{0}^{1}-n_{0}^{2}\left\|_{\mathrm{TV}}+\int_{0}^{t}\right\|\left(p\left(N_{1}(\tau), \cdot\right)-p\left(N_{2}(\tau), \cdot\right)\right) n_{1}(\tau, \cdot) \|_{\mathrm{TV}} \mathrm{d} \tau \\
& +\int_{0}^{t}\left\|p\left(N_{2}(\tau), \cdot\right)\left(n_{1}(\tau)-n_{2}(\tau)\right)\right\|_{\mathrm{TV}} \mathrm{d} \tau+\int_{0}^{t}\left\|\left(N_{1}(\tau)-N_{2}(\tau)\right) \delta_{0}\right\|_{\mathrm{TV}} \mathrm{d} \tau
\end{aligned}
$$

and with very similar arguments as before we obtain that

$$
\begin{array}{r}
\left\|n_{1}(t)-n_{2}(t)\right\|_{\mathrm{TV}} \leq\left\|n_{0}^{1}-n_{0}^{2}\right\|_{\mathrm{TV}}+2 L\left\|n_{0}\right\|_{\mathrm{TV}}\|p\|_{\infty} \int_{0}^{t}\left\|n_{1}(\tau)-n_{2}(\tau)\right\|_{\mathrm{TV}} \mathrm{d} \tau \\
+\|p\|_{\infty} \int_{0}^{t}\left\|n_{1}(\tau)-n_{2}(\tau)\right\|_{\mathrm{TV}} \mathrm{d} \tau+2\|p\|_{\infty} \int_{0}^{t}\left\|n_{1}(\tau)-n_{2}(\tau)\right\|_{\mathrm{TV}} \mathrm{d} \tau \\
\leq\left\|n_{0}^{1}-n_{0}^{2}\right\|_{\mathrm{TV}}+4\|p\|_{\infty} \int_{0}^{t}\left\|n_{1}(\tau)-n_{2}(\tau)\right\|_{\mathrm{TV}} \mathrm{d} \tau .
\end{array}
$$

Gronwall's inequality then implies (22).

Weak solutions Definition 3.2 is convenient for finding solutions, but later we will need a more manageable form:

Definition 3.8. (Weak solution to (1)) Assume $p$ satisfies (5) and is nonnegative. A couple of functions $n \in \mathcal{C}\left([0, T), \mathcal{M}_{+}\left(\mathbb{R}_{0}^{+}\right)\right)$and $N \in \mathcal{C}([0, T),[0,+\infty))$, defined on an interval $[0, T)$ for some $T \in(0,+\infty]$, is called a weak measure solution to (1) with 
initial data $n_{0} \in \mathcal{M}\left(\mathbb{R}_{0}^{+}\right)$and $N_{0} \in \mathbb{R}$ if it satisfies $n(0)=n_{0}, N(0)=N_{0}$, and for each $\varphi \in \mathcal{C}_{\mathrm{c}}^{\infty}(0,+\infty)$ the function $t \mapsto \int_{0}^{\infty} \varphi(s) n(t, s) \mathrm{d} s$ is absolutely continuous and

$$
\begin{aligned}
& \frac{\mathrm{d}}{\mathrm{d} t} \int_{0}^{\infty} \varphi(s) n(t, s) \mathrm{d} s \\
& \quad=\int_{0}^{\infty} \partial_{s} \varphi(s) n(t, s) \mathrm{d} s-\int_{0}^{\infty} p(N(t), s) n(t, s) \varphi(s) \mathrm{d} s+\int_{0}^{\infty} N(t) \delta_{0}(s) \varphi(s) \mathrm{d} s .
\end{aligned}
$$

for almost all $t \in[0, T)$, and

$$
N(t)=\int_{0}^{\infty} p(N(t), s) n(t, s) \mathrm{d} s, \quad \text { for all } t \in[0, T) .
$$

Equivalence results between definitions based on the Duhamel formula and definitions of weak solutions based on integration against a test function are fairly common. Here we use the main theorem in Ball (1977) with $f(t, \cdot)=-p(N(t), \cdot) n(t, \cdot)-N(t) \delta_{0}(\cdot)$, which implies that mild solutions of our equation are are equivalent to weak solutions:

Theorem 3.9 (Ball (1977)). Assume p satisfies (5) and is nonnegative, and take $T \in$ $(0,+\infty]$. A function $n:[0, T) \rightarrow \mathcal{M}_{+}\left(\mathbb{R}_{0}^{+}\right)$is a weak measure solution (cf. Definition 3.8) to (1) if and only if it is a mild measure solution (cf. Definition 3.2).

\subsection{The linear equation}

When $p=p(N, s)$ does not depend on $N$, equation (1) becomes linear:

$$
\left\{\begin{array}{l}
\frac{\partial}{\partial t} n(t, s)+\frac{\partial}{\partial s} n(t, s)+p(s) n(t, s)=0, \quad t, s>0 \\
N(t):=n(t, s=0)=\int_{0}^{+\infty} p(s) n(t, s) \mathrm{d} s, \quad t>0 \\
n(t=0, s)=n_{0}(s), \quad s \geq 0 .
\end{array}\right.
$$

This is referred to as the "no-connectivity" case or the " $J=0$ case" in Pakdaman et al. (2010).

\subsubsection{Well-posedness}

We give a similar definition for mild measure solutions:

Definition 3.10. Assume $p:[0,+\infty) \rightarrow[0,+\infty)$ is a bounded measurable function. A function $n \in \mathcal{C}\left([0, T), \mathcal{M}_{+}\left(\mathbb{R}_{0}^{+}\right)\right)$, defined on an interval $[0, T)$ for some $T \in(0,+\infty]$, is called a mild measure solution to (32) with initial data $n_{0} \in \mathcal{M}\left(\mathbb{R}_{0}^{+}\right)$if it satisfies $n(0)=n_{0}$ and

$$
n(t, s)=T_{t} n_{0}(s)-\int_{0}^{t} T_{t-\tau}(p(\cdot) n(\tau, \cdot))(s) \mathrm{d} \tau+\int_{0}^{t} T_{t-\tau}\left(N(\tau) \delta_{0}\right)(s) \mathrm{d} \tau
$$

for all $t \in[0, T)$, with

$$
N(t):=\int_{0}^{\infty} p(s) n(t, s) \mathrm{d} s, \quad t \in[0, T) .
$$


Our existence result stated in 3.6 easily gives the following as a consequence:

Theorem 3.11 (Well-posedness of (32) in measures). Assume that $p:[0,+\infty) \rightarrow$ $[0,+\infty)$ is bounded, Lipschitz and nonnegative. For any given initial data $n_{0} \in \mathcal{M}\left(\mathbb{R}^{+}\right)$ there exists a unique measure solution $n \in \mathcal{C}\left([0,+\infty) ; \mathcal{M}\left(\mathbb{R}_{0}^{+}\right)\right)$of the linear equation (32) in the sense of Definition 3.10. In addition, if $n$ is any mild measure solution to (32) defined on any interval $[0, T)$ then

$$
\|n(t)\|_{\mathrm{TV}} \leq\|n(0)\|_{\mathrm{TV}} \quad \text { for all } t \in[0, T) .
$$

Proof. This result can be mostly deduced from Theorem 3.6. For the existence part, split $n_{0}$ into its positive and negative parts as $n_{0}=n_{0}^{+}-n_{0}^{-}$. Theorem 3.6 gives the existence of two solutions $n^{+}$and $n^{-}$with initial data $n_{0}^{+}$and $n_{0}^{-}$, respectively; then $n:=n^{+}-n^{-}$is a mild measure solution with initial data $n_{0}$. For uniqueness, if $n$ is any mild solution on $[0, T)$, the same argument as in (29)-(30) shows that

$$
\|n(t)\|_{\mathrm{TV}} \leq\left\|n_{0}\right\|_{\mathrm{TV}}+4\|p\|_{\infty} \int_{0}^{t}\left\|n_{1}(\tau)\right\|_{\mathrm{TV}} \mathrm{d} \tau,
$$

which gives by Gronwall's inequality that

$$
\|n(t)\|_{\mathrm{TV}} \leq\left\|n_{0}\right\|_{\mathrm{TV}} e^{4\|p\|_{\infty} t} \quad \text { for all } t \in[0, T) .
$$

In particular, by linearity this implies solutions are unique. Finally, with the same argument as in Lemma 3.4 one sees that for any solution $n$ defined on $[0, T)$ it holds

$$
\int_{\mathbb{R}_{0}^{+}} n(t, s) \mathrm{d} s=\int_{\mathbb{R}_{0}^{+}} n_{0}(s) \mathrm{d} s
$$

for all $t \in[0, T)$. Due to uniqueness, with the same splitting we used at the beginning of the proof we have $n(t)=n^{+}(t)-n^{-}(t)$, so

$$
\|n(t)\|_{\mathrm{TV}} \leq\left\|n^{+}(t)\right\|_{\mathrm{TV}}+\left\|n^{-}(t)\right\|_{\mathrm{TV}}=\left\|n_{0}^{+}\right\|_{\mathrm{TV}}+\left\|n_{0}^{-}\right\|_{\mathrm{TV}}=\left\|n_{0}\right\|_{\mathrm{TV}}
$$

which finishes the proof.

The above result allows us to define an evolution semigroup $\left(S_{t}\right)_{t \geq 0}$ (in fact it is a $C_{0}$-semigroup on $\mathcal{M}$ with the bounded Lipschitz topology) by setting

$$
S_{t}: \mathcal{M} \rightarrow \mathcal{M}, \quad S_{t}\left(n_{0}\right):=n(t)
$$

for any $n_{0} \in \mathcal{M}$, where $n(t)$ is the mild measure solution to (32) with initial data $n_{0}$.

Stationary solutions for the linear equation We remark that Theorem 3.15 below implies that the linear equation (32) has a unique stationary solution in the space of probabilities on $[0,+\infty$ ) (for $p$ bounded, Lipschitz, and satisfying (8)); of course in this case this solution is explicit, given by

$$
n_{*}(s):=N_{*} e^{-\int_{0}^{s} p(\tau) \mathrm{d} \tau}, \quad s \geq 0,
$$

where $N_{*}$ is the appropriate normalisation constant that makes this a probability density. Although Theorem 3.15 does not rule out the existence of other stationary solutions which may not be probabilities, since the solution is explicit it is not difficult to see that, up to a constant factor, $n_{*}$ is the only stationary solution within the set of all finite measures. This is also a consequence of Doeblin's theorem below. 


\subsubsection{Positive lower bound}

Our main result on the spectral gap for the linear operator is based on the fact that for any initial probability distribution, solutions have a universal lower bound after a fixed time. We give the following lemma:

Lemma 3.12. Let $p:[0,+\infty) \rightarrow[0,+\infty)$ be bounded, Lipschitz function satisfying (7) and (8), and consider the semigroup $\left(S_{t}\right)_{t \geq 0}$ given by the existence Theorem 3.11. Then $S_{t_{0}}$ satisfies Doeblin's condition (12) for $t_{0}=2 s_{*}$ and $\alpha=p_{\min } s_{*} e^{-2 p_{\max } s_{*}}$. More precisely, for $t_{0}=2 s_{*}$ we have

$$
S_{2 s_{*}} n_{0}(s) \geq p_{\min } e^{-2 p_{\max } s_{*}} \mathbb{1}_{\left\{0<s<s_{*}\right\}}
$$

for all probability measures $n_{0}$ on $[0,+\infty)$.

Proof. We define a semigroup $\tilde{S}_{t}$ associated to the linear problem

$$
\left\{\begin{array}{l}
\frac{\partial}{\partial t} \tilde{n}(t, s)+\frac{\partial}{\partial s} \tilde{n}(t, s)=-p(s) \tilde{n}(t, s), \quad t, s>0 \\
\tilde{n}(t, 0)=0, \quad t>0 \\
\tilde{n}(0, s)=n_{0}(s), \quad s \geq 0
\end{array}\right.
$$

which has the explicit solution

$$
\tilde{n}(t, s)= \begin{cases}n_{0}(s-t) e^{-\int_{0}^{t} p(s-t+\tau) \mathrm{d} \tau}, & s>t, \\ 0, & t>s .\end{cases}
$$

Then we write the solution to the linear equation (32) as

$$
n(t, s)=\tilde{S}_{t} n_{0}(s)+\int_{0}^{t} \tilde{S}_{t-\tau}\left(N(\tau) \delta_{0}\right)(s) \mathrm{d} \tau
$$

For $s>t$ we have

$$
\begin{aligned}
& n(t, s) \geq \tilde{S}_{t} n_{0}(s)=n_{0}(s-t) e^{-\int_{0}^{t} p(s-t+\tau) \mathrm{d} \tau} \geq n_{0}(s-t) e^{-p_{\max } t} \\
& \tilde{S}_{t-\tau} n_{0}(s) \geq n_{0}(s-t+\tau) e^{-p_{\max }(t-\tau)}
\end{aligned}
$$

Then for $t>s_{*}$ it holds that

$$
\begin{aligned}
N(t)=\int_{0}^{+\infty} p(s) n(t, s) \mathrm{d} s \geq p_{\min } e^{-p_{\max } t} & \int_{s_{*}}^{\infty} n_{0}(s-t) \mathrm{d} s \\
& \geq p_{\min } e^{-p_{\max } t} \int_{0}^{+\infty} n_{0}(s) \mathrm{d} s=p_{\min } e^{-p_{\max } t} .
\end{aligned}
$$

Therefore, for any $s>0$ and $t>s+s_{*}$ we have:

$$
\begin{aligned}
n(t, s) \geq \int_{0}^{t} \tilde{S}_{t-\tau}\left(N(\tau) \delta_{0}\right)(s) \mathrm{d} \tau \geq \int_{s_{*}}^{t} \tilde{S}_{t-\tau}\left(p_{\min } e^{-p_{\max } \tau} \delta_{0}\right)(s) \mathrm{d} \tau \\
\quad \geq p_{\min } \int_{s_{*}}^{t} e^{-p_{\max } \tau} e^{-p_{\max }(t-\tau)} \delta_{0}(s-t+\tau) \mathrm{d} \tau=p_{\min } e^{-p_{\max } t} \mathbb{1}_{\left\{0<s<t-s_{*}\right\}} .
\end{aligned}
$$

Hence for $t=2 s_{*}$ and all $0<s<s_{*}$ we obtain the result. 


\subsubsection{Spectral gap}

Exponential convergence to the equilibrium for the linear equation is an immediate consequence of Theorem 2.3. We give the following proposition based on that:

Proposition 3.13. For a given initial data $n_{0} \in \mathcal{M}\left(\mathbb{R}^{+}\right)$, let $p:[0, \infty) \rightarrow[0,+\infty)$ be bounded, Lipschitz function satisfying (7) and (8). Then, there exists a unique probability measure $n_{*} \in \mathcal{P}([0,+\infty))$ which is a stationary solution to $(32)$, and any other stationary solution is a multiple of it. Also, for

$$
C=\frac{1}{1-\alpha}>1 \text { and } \lambda:=-\frac{\log (1-\alpha)}{t_{0}}
$$

we have

$$
\left\|S_{t}\left(n_{0}-n_{*}\right)\right\|_{\mathrm{TV}} \leq C e^{-\lambda t}\left\|n_{0}-n_{*}\right\|_{\mathrm{TV}}, \text { for all } t \geq 0 .
$$

In addition, for $t_{0}:=2 s_{*}$ we have

$$
\left\|S_{t_{0}}\left(n_{1}-n_{2}\right)\right\|_{\mathrm{TV}} \leq(1-\alpha)\left\|n_{1}-n_{2}\right\|_{\mathrm{TV}}
$$

for any probability distributions $n_{1}, n_{2}$, and with

$$
\alpha:=p_{\min } s_{*} e^{-2 p_{\max } s_{*}} .
$$

Proof. We apply Theorem 2.3, since Lemma 3.12 shows that $S_{t_{0}}$ satisfies the Doeblin condition for $t_{0}=2 s_{*}$. Moreover,

$$
C=\frac{1}{1-\alpha}=e^{\lambda t_{0}}=e^{-\log \left(1-p_{\min } s_{*} e^{-2 p_{\max } s_{*}}\right)}=\frac{1}{1-p_{\min } s_{*} e^{-2 p_{\max } s_{*}}}>1 .
$$

\subsection{Stationary solutions for the nonlinear equation}

Definition 3.14. We say that a nonnegative function $n_{*} \in \mathcal{C}([0,+\infty)) \cap \mathcal{C}^{1}(0,+\infty)$ is a stationary solution to (1) if it satisfies

$$
\left\{\begin{array}{l}
\frac{\partial}{\partial s} n_{*}(s)+p\left(N_{*}, s\right) n_{*}(s)=0, \quad s>0, \\
n_{*}(0)=: N_{*}=\int_{0}^{+\infty} p\left(N_{*}, s\right) n_{*}(s) \mathrm{d} s .
\end{array}\right.
$$

The following result is essentially the same as that given in Pakdaman et al. (2010). There it is proved for a particular form of $p$, i.e. for $p(x, s)=\mathbb{1}_{s>s^{*}(x)}$, for some nonnegative $s^{*} \in C^{1}([0,+\infty))$ such that $\frac{d}{d x} s^{*}(x) \leq 0$ with $s^{*}(0)<1$. So we prove it here for completeness, and to adapt it to our precise assumptions:

Theorem 3.15. Assume (5), (6), (8) for $p$ and also that

$$
L<\left(p_{\max }\right)^{-2}\left(\frac{s_{*}^{2}}{2}+\frac{s_{*}}{p_{\min }}+\frac{1}{p_{\min }^{2}}\right)^{-1} .
$$

Then there exists a unique probability measure $n_{*}$ which is a stationary solution to (1). 
Proof. If there is a stationary solution $n_{*}$ then (since the first equation of (39) is an ordinary differential equation) it must satisfy

$$
n_{*}(s)=n_{*}(0) e^{-\int_{0}^{s} p\left(N_{*}, \tau\right) \mathrm{d} \tau}=N_{*} e^{-\int_{0}^{s} p\left(N_{*}, \tau\right) \mathrm{d} \tau}, \quad s \geq 0 .
$$

If $n_{*}$ is a probability, by integrating we see that

$$
N_{*}=\left(\int_{0}^{+\infty} e^{-\int_{0}^{s} p\left(N_{*}, \tau\right) \mathrm{d} \tau} \mathrm{d} s\right)^{-1} .
$$

In particular, $N_{*}$ must be strictly positive. Conversely, if $N_{*}>0$ is such that (41) is satisfied then we may define $n_{*}=n_{*}(s)$ by $(40)$ and it is straightforward to check that it is a probability, and it is a stationary solution to (1). Hence the problem is reduced to showing that there exists a unique solution $N_{*}>0$ to (41); this is ensured by a simple fixed point argument, since

$$
\begin{gathered}
\frac{\partial}{\partial N}\left(\int_{0}^{+\infty} e^{-\int_{0}^{s} p(N, \tau) \mathrm{d} \tau} \mathrm{d} s\right)^{-1}=\frac{\int_{0}^{+\infty}\left(\int_{0}^{s} \partial_{N} p(N, \tau) d \tau\right)\left(e^{-\int_{0}^{s} p(N, \tau) \mathrm{d} \tau}\right) \mathrm{d} s}{\left(\int_{0}^{+\infty} e^{-\int_{0}^{s} p(N, \tau) \mathrm{d} \tau} \mathrm{d} s\right)^{2}} \\
\leq L \frac{\int_{0}^{+\infty} s e^{-\int_{0}^{s} p(N, \tau) \mathrm{d} \tau} \mathrm{d} s}{\left(\int_{0}^{+\infty} e^{-p_{\max } s} \mathrm{~d} s\right)^{2}} \leq L\left(p_{\max }\right)^{2}\left(\int_{0}^{s_{*}} s \mathrm{~d} s+\int_{s_{*}}^{+\infty} s e^{-p_{\min }\left(s-s_{*}\right)} \mathrm{d} s\right) \\
=L\left(p_{\max }\right)^{2}\left(\frac{s_{*}^{2}}{2}+\frac{s_{*}}{p_{\min }}+\frac{1}{p_{\min }^{2}}\right)<1 .
\end{gathered}
$$

where we have used (6) and (8). Note that these calculation is rigorous due to (5) and the fact that the integrals in $s$ converge uniformly for all $N$.

Similarly to our main results, the condition on $L$ in the above theorem can be understood as a condition of weak nonlinearity.

\subsection{Asymptotic behaviour}

In this section we prove Theorem 1.1 for equation (1). Formally, the proof is based on rewriting it as

$$
\frac{\partial}{\partial t} n=\mathcal{L}_{N}(n)=\mathcal{L}_{N_{*}}(n)+\left(\mathcal{L}_{N}(n)-\mathcal{L}_{N_{*}}(n)\right)=: \mathcal{L}_{N_{*}}(n)+h
$$

where we define

$$
\mathcal{L}_{N}(n)(t, s):=-\frac{\partial}{\partial s} n(t, s)-p(N(t), s) n(t, s)+\delta_{0}(s) \int_{0}^{\infty} p(N(t), u) n(t, u) \mathrm{d} u,
$$

and

$$
\begin{aligned}
h(t, s):=\left[p\left(N_{*}, s\right)-p(N(t), s)\right] & n(t, s) \\
& +\delta_{0}(s) \int_{0}^{+\infty}\left[p(N(t), u)-p\left(N_{*}, u\right)\right] n(t, u) \mathrm{d} u .
\end{aligned}
$$


We treat the term $h$ as a perturbation. In order to do this rigorously, notice that $h$ contains a multiple of $\delta_{0}$, so it is necessary to use a concept of solution in a space of measures. Then, since the solutions we are using do not allow us to write (42) rigorously, we need to use a concept of solution that allows for the same formal computation; this is the reason why weak solutions were introduced earlier.

Before proving the Theorem 1.1 for equation (1) we need the following lemma:

Lemma 3.16. Assume the conditions in Theorem 1.1 for equation (1). Then $h$, defined by (43), satisfies

$$
\|h(t)\|_{\mathrm{TV}} \leq \tilde{C}\left\|n(t)-n_{*}\right\|_{\mathrm{TV}} \quad \text { for all } t \geq 0,
$$

where $\tilde{C}:=2 p_{\max } \frac{L}{1-L}$. It also satisfies

$$
\int_{0}^{\infty} h(t, s) \mathrm{d} s=0 \quad \text { for all } t \geq 0 .
$$

Proof. We notice that the stationary solution $n_{*}$ exists due to Theorem 3.15, and the solution $n(t) \equiv n(t, s)$ with initial data $n_{0}$ was obtained in Theorem 3.6. Call $N_{*}$ the total firing rate corresponding to the stationary solution $n_{*}$. We estimate directly each of the terms in the expression of $h$ :

$$
\begin{gathered}
\|h(t)\|_{\mathrm{TV}} \leq\left\|\left(p\left(N_{*}, s\right)-p(N(t), s)\right) n(t, s)\right\|_{\mathrm{TV}}+\left\|\delta_{0} \int_{0}^{+\infty}\left(p(N(t), s)-p\left(N_{*}, s\right)\right) n(t, s) \mathrm{d} s\right\|_{\mathrm{TV}} \\
\leq\left\|p\left(N_{*}, s\right)-p(N(t), s)\right\|_{\infty}\|n(t)\|_{\mathrm{TV}}+\left|\int_{0}^{+\infty}\left(p(N(t), s)-p\left(N_{*}, s\right)\right) n(t, s) \mathrm{d} s\right| \\
\leq L\left|N_{*}-N(t)\right|+\left\|p\left(N_{*}, s\right)-p(N(t), s)\right\|_{\infty}\|n(t)\|_{\mathrm{TV}} \\
\leq \frac{L p_{\max }}{1-L}\left\|n(t)-n_{*}\right\|_{\mathrm{TV}}+L\left|N_{*}-N(t)\right| \leq 2 p_{\max } \frac{L}{1-L}\left\|n(t)-n_{*}\right\|_{\mathrm{TV}},
\end{gathered}
$$

where the last inequality is due to Lemma 3.5 and the fact that $\left\|n_{*}\right\|_{\mathrm{TV}}=\|n(t)\|_{\mathrm{TV}}=1$, which imply

$$
\left|N_{*}-N(t)\right| \leq \frac{p_{\max }}{1-L}\left\|n(t)-n_{*}\right\|_{\mathrm{TV}} .
$$

Regarding the integral of $h$ in $s$ we have

$$
\begin{aligned}
\int_{0}^{+\infty} h(t, s) \mathrm{d} s & =\int_{0}^{+\infty}\left[p\left(N_{*}, s\right)-p(N(t), s)\right] n(t, s) \mathrm{d} s \\
& +\int_{0}^{+\infty} \delta_{0}(x) \int_{0}^{+\infty}\left[p(N(t), s)-p\left(N_{*}, s\right)\right] n(t, s) \mathrm{d} s \mathrm{~d} x \\
& =\int_{0}^{+\infty}\left[p\left(N_{*}, s\right)-p(N(t), s)\right] n(t, s) \mathrm{d} s+\int_{0}^{+\infty}\left[p(N(t), s)-p\left(N_{*}, s\right)\right] n(t, s) \mathrm{d} s \\
& =0
\end{aligned}
$$

which gives the result.

Proof of Theorem 1.1 for eq. (1). Call $N_{*}$ the value of the total firing rate at equilibrium. The solution $n$ to equation (1) is in particular a weak solution (see Theorem 3.9). Then one sees it is also a weak solution (in the sense of Ball (1977)) to the equation

$$
\frac{\mathrm{d}}{\mathrm{d} t} n(t, \cdot)=\mathcal{L}_{N_{*}} n(t, \cdot)+h(t, \cdot),
$$


where $\mathcal{L}_{N_{*}}$ is the linear operator corresponding to $p=p\left(N_{*}, s\right)$ for $N_{*}$ fixed,

$$
\mathcal{L}_{N_{*}} n(t, s):=-\frac{\partial}{\partial s} n(t, s)-p\left(N_{*}, s\right) n(t, s)+\delta_{0} \int_{0}^{+\infty} p\left(N_{*}, u\right) n(t, u) \mathrm{d} u .
$$

Then by Ball (1977) we may use Duhamel's formula and write the solution as

$$
n(t, s)=S_{t} n_{0}(s)+\int_{0}^{t} S_{t-\tau} h(\tau, s) \mathrm{d} \tau
$$

where $S_{t}$ is the linear semigroup defined in Section 3.2. We subtract the stationary solution from both sides;

$$
n(t, s)-n_{*}(s)=S_{t} n_{0}(s)-n_{*}(s)+\int_{0}^{t} S_{t-\tau} h(\tau, s) \mathrm{d} \tau .
$$

Then we take the TV norm;

$$
\left\|n(t)-n_{*}\right\|_{\mathrm{TV}} \leq\left\|S_{t} n_{0}-n_{*}\right\|_{\mathrm{TV}}+\left\|\int_{0}^{t} S_{t-\tau} h(\tau, s) \mathrm{d} \tau\right\|_{\mathrm{TV}} .
$$

By using Lemma 3.16 and Proposition 3.13, Equation (46) becomes:

$$
\begin{aligned}
\left\|n(t)-n_{*}\right\|_{\mathrm{TV}} & \leq\left\|S_{t}\left(n_{0}-n_{*}\right)\right\|_{\mathrm{TV}}+\int_{0}^{t}\left\|S_{t-\tau} h(\tau, s)\right\|_{\mathrm{TV}} \mathrm{d} \tau \\
& \leq C e^{-\lambda t}\left\|n_{0}-n_{*}\right\|_{\mathrm{TV}}+\tilde{C} \int_{0}^{t} e^{-\lambda(t-\tau)}\left\|n(\tau)-n_{*}\right\|_{\mathrm{TV}} \mathrm{d} \tau .
\end{aligned}
$$

Therefore, by Gronwall's inequality we obtain

$$
\left\|n(t)-n_{*}\right\|_{\mathrm{TV}} \leq C e^{-(\lambda-\tilde{C}) t}\left\|n_{0}-n_{*}\right\|_{\mathrm{TV}} .
$$

\section{A structured neuron population model with fatigue}

We now consider Equation (3) for a structured neuron population model. We follow the same order as in Section 3.

\subsection{Well-posedness}

We refer the reader to Section 3.1 for preliminary notation and useful results. We define mild measure solutions in a similar way. Still denoting by $\left(T_{t}\right)_{t \geq 0}$ the translation semigroup generated on $\left(\mathcal{M},\|\cdot\|_{\mathrm{BL}}\right)$ by the operator $-\frac{\partial}{\partial s}$, we rewrite (3) as

$$
\frac{\partial}{\partial t} n(t, s)-\mathcal{L} n(t, s)=A[n](t, s)
$$

where

$$
\mathcal{L}=-\frac{\partial}{\partial s} \quad \text { and } \quad A[n](t, s):=-p(N(t), s) n(t, s)+\int_{0}^{+\infty} \kappa(s, u) p(N(t), u) n(t, u) \mathrm{d} u .
$$


Definition 4.1. Assume that $p$ satisfies (5),(6) and $\kappa$ satisfies (9). A couple of functions $n \in \mathcal{C}\left([0, T), \mathcal{M}_{+}\left(\mathbb{R}_{0}^{+}\right)\right)$and $N \in \mathcal{C}([0, T),[0,+\infty))$, defined on an interval $[0, T)$ for some $T \in(0,+\infty)$, is called a mild measure solution to $(3)$ with initial data $n_{0}(s) \in$ $\mathcal{M}\left(\mathbb{R}_{0}^{+}\right), n(0)=n_{0}$ if it satisfies

$$
n(t, s)=T_{t} n_{0}(s)+\int_{0}^{t} T_{t-\tau} A[n(\tau, \cdot)](s) d \tau, \quad \text { for all } t \in[0, T),
$$

where $A[n](t, s)$ is defined as in (47) and

$$
N(t)=\int_{0}^{+\infty} p(N(t), s) n(t, s) d s, \quad t \in[0, T)
$$

By integrating in $\mathbb{R}_{0}^{+}$, Definition 4.1 directly implies mass conservation. Therefore Lemma 3.4 holds true for this equation as well. Moreover we have the Lemma 3.5 satisfied with the same constants.

Theorem 4.2 (Well-posedness of (3) in measures). Assume that $p$ satisfies (5), (6) and the Lipschitz constant $L$ in (6) satisfies $L \leq 1 /\left(4\left\|n_{0}\right\|_{\mathrm{TV}}\right)$. Assume also (9) for $\kappa$. For any given initial data $n_{0} \in \mathcal{M}\left(\mathbb{R}^{+}\right)$there exists a unique measure solution $n \in \mathcal{C}\left([0,+\infty) ; \mathcal{M}\left(\mathbb{R}_{0}^{+}\right)\right)$of $(3)$ in the sense of Definition 4.1. In addition, if $n_{1}, n_{2}$ are any two mild measure solutions to (1) (with possibly different initial data) defined on any interval $[0, T)$ then

$$
\left\|n_{1}(t)-n_{2}(t)\right\|_{\mathrm{TV}} \leq\left\|n_{1}(0)-n_{2}(0)\right\|_{\mathrm{TV}} e^{4\|p\|_{\infty} t} \quad \text { for all } t \in[0, T) .
$$

Proof of Theorem 4.2. Let us first show existence of a solution for a nonnegative initial measure $n_{0} \in \mathcal{M}_{+}\left(\mathbb{R}_{0}^{+}\right)$. If $n_{0}=0$ it is clear that setting $n(t)$ equal to the zero measure on $\mathbb{R}_{0}^{+}$for all $t$ defines a solution, so we assume $n_{0} \neq 0$. Fix $C, T>0$, to be chosen later. Consider the complete metric space

$$
\mathcal{Y}=\left\{n \in \mathcal{C}\left([0, T], \mathcal{M}_{+}\left(\mathbb{R}_{0}^{+}\right)\right) \mid n(0)=n_{0},\|n(t)\|_{\mathrm{TV}} \leq C \text { for all } t \in[0, T]\right\},
$$

endowed with the norm

$$
\|n\|_{\mathcal{Y}}:=\sup _{t \in[0, T]}\|n(t)\|_{\mathrm{TV}}
$$

We remark that $\mathcal{C}\left([0, T], \mathcal{M}\left(\mathbb{R}_{0}^{+}\right)\right)$refers to functions which are continuous in the bounded Lipschitz topology, not in the total variation one. Define an operator $\Gamma: \mathcal{Y} \rightarrow \mathcal{Y}$ by

$$
\Gamma[n](t):=T_{t} n_{0}+\int_{0}^{t} T_{t-\tau}(A[n](\tau,,))(s) d \tau
$$

for all $n \in \mathcal{Y}$.

The definition of $\Gamma[n]$ indeed makes sense, since $\tau \mapsto T_{t-\tau}(A[n](\tau, \cdot))$ is a continuous function from $[0, T]$ to $\mathcal{M}\left(\mathbb{R}_{0}^{+}\right)$, hence integrable.

We first check that $\Gamma[n]$ is indeed in $\mathcal{Y}$. It is easy to see that $t \mapsto \Gamma[n](t)$ is continuous in the bounded Lipschitz topology, and it is a nonnegative measure for each $t \in[0, T]$. 
We also have

$$
\begin{aligned}
&\|\Gamma[n](t)\|_{\mathrm{TV}} \leq\left\|n_{0}\right\|_{\mathrm{TV}}+\int_{0}^{t}\|p(N(\tau), \cdot) n(\tau, \cdot)\|_{\mathrm{TV}} \mathrm{d} \tau \\
&+\int_{0}^{t}\left\|\int_{0}^{+\infty} \kappa(., u) p(N(\tau), u) n(\tau, u) \mathrm{d} u\right\|_{\mathrm{TV}} \mathrm{d} \tau \\
&+\int_{0}^{t} \int_{0}^{+\infty}\left|\left(\int_{0}^{+\infty} \kappa(s, u) \mathrm{d} s\right) p(N(\tau), u) n(\tau, u)\right| \mathrm{d} u \mathrm{~d} \tau \\
& \leq\left\|n_{0}\right\|_{\mathrm{TV}}+T C\|p\|_{\infty}+\int_{0}^{t}\|p(N(\tau), \cdot) n(\tau, \cdot)\|_{\mathrm{TV}} \mathrm{d} \tau \\
& \leq\left\|n_{0}\right\|_{\mathrm{TV}}+2 T C\left\|_{\infty}\right\|_{\infty}
\end{aligned}
$$

Here we used the assumption (9) on $\kappa$. We choose

$$
T \leq \frac{1}{4\|p\|_{\infty}}, \quad \text { and } \quad C:=2\left\|n_{0}\right\|_{\mathrm{TV}}
$$

so that

$$
\left\|n_{0}\right\|_{\mathrm{TV}}+2 T C\|p\|_{\infty} \leq\left\|n_{0}\right\|_{\mathrm{TV}}+\frac{C}{2} \leq C .
$$

Hence with these conditions on $T$ and $C$ we have $\Gamma[n] \in \mathcal{Y}$.

Let us show that $\Gamma$ is a contraction mapping. Take $n_{1}, n_{2} \in \mathcal{Y}$ and let $N_{1}, N_{2}$ be defined by (24) corresponding to $n_{1}$ and $n_{2}$, respectively. We have

$$
\begin{gathered}
\left\|\Gamma\left[n_{1}\right](t)-\Gamma\left[n_{2}\right](t)\right\|_{\mathrm{TV}} \leq \int_{0}^{t}\left\|\left(p\left(N_{1}(\tau), \cdot\right)-p\left(N_{2}(\tau), \cdot\right)\right) n_{1}(\tau, \cdot)\right\|_{\mathrm{TV}} \mathrm{d} \tau \\
\quad+\int_{0}^{t}\left\|p\left(N_{2}(\tau), \cdot\right)\left(n_{1}(\tau, \cdot)-n_{2}(\tau, \cdot)\right)\right\|_{\mathrm{TV}} \mathrm{d} \tau \\
+\int_{0}^{t}\left\|\int_{0}^{+\infty} \kappa(\cdot, u)\left(p\left(N_{1}(\tau), u\right)-p\left(N_{2}(\tau), u\right)\right) n_{1}(\tau, u) \mathrm{d} u\right\|_{\mathrm{TV}} \mathrm{d} \tau \\
+\int_{0}^{t}\left\|\int_{0}^{+\infty} \kappa(\cdot, u) p\left(N_{2}(\tau), u\right)\left(n_{1}(\tau, u)-n_{2}(\tau, u)\right) \mathrm{d} u\right\|_{\mathrm{TV}} \mathrm{d} \tau \\
=: T_{1}+T_{2}+T_{3}+T_{4} .
\end{gathered}
$$

We bound each term separately. We can bound $T_{3}$ and $T_{4}$ in the following way;

$$
\begin{aligned}
& T_{3} \leq \int_{0}^{t} \int_{0}^{+\infty}\left|\left(\int_{0}^{+\infty} \kappa(s, u) \mathrm{d} s\right)\left(p\left(N_{1}(\tau), u\right)-p\left(N_{2}(\tau), u\right)\right) n_{1}(\tau, u)\right| \mathrm{d} u \mathrm{~d} \tau=T_{1}, \\
& T_{4} \leq \int_{0}^{t} \int_{0}^{+\infty}\left|\left(\int_{0}^{+\infty} \kappa(s, u) \mathrm{d} s\right) p\left(N_{2}(\tau), \cdot\right)\left(n_{1}(\tau)-n_{2}(\tau)\right)\right| \mathrm{d} u \mathrm{~d} \tau=T_{2}
\end{aligned}
$$

For $T_{1}$, since $L \leq 1 /\left(4\left\|n_{0}\right\|_{\mathrm{TV}}\right)$, using Lemma 3.5 as previously calculated we have

$$
T_{1} \leq T\|p\|_{\infty}\left\|n_{1}-n_{2}\right\|_{\mathcal{Y}},
$$

and for $T_{2}$,

$$
T_{2} \leq T\|p\|_{\infty}\left\|n_{1}-n_{2}\right\|_{\mathcal{Y}} .
$$


Putting equations (52)-(53) together and taking the supremum over $0 \leq t \leq T$,

$$
\left\|\Gamma\left[n_{1}\right]-\Gamma\left[n_{2}\right]\right\|_{\mathcal{Y}} \leq 4 T\|p\|_{\infty}\left\|n_{1}-n_{2}\right\|_{\mathcal{Y}}
$$

Taking now $T \leq 1 /\left(4\|p\|_{\infty}\right)$ ensures that $\Gamma$ is contractive, so it has a unique fixed point in $\mathcal{Y}$, which is a mild measure solution on $[0, T]$. If we call $n$ this fixed point, since $\|n(T)\|_{\mathrm{TV}}=\left\|n_{0}\right\|_{\mathrm{TV}}$ by mass conservation (see Lemma 3.4), we may repeat this argument to continue the solution on $[T, 2 T],[2 T, 3 T]$, showing that there is a solution defined on $[0,+\infty)$.

In order to show stability of solutions with respect to the initial data (which implies uniqueness of solutions), take two measures $n_{0}^{1}, n_{0}^{2} \in \mathcal{M}_{+}\left(\mathbb{R}_{0}^{+}\right)$, and consider two solutions $n_{1}, n_{2}$ with initial data $n_{0}^{1}, n_{0}^{2}$ respectively. We have

$$
\begin{gathered}
\left\|n_{1}(t)-n_{2}(t)\right\|_{\mathrm{TV}} \leq\left\|n_{0}^{1}-n_{0}^{2}\right\|_{\mathrm{TV}}+\int_{0}^{t}\left\|\left(p\left(N_{1}(\tau), \cdot\right)-p\left(N_{2}(\tau), \cdot\right)\right) n_{1}(\tau, \cdot)\right\|_{\mathrm{TV}} \mathrm{d} \tau \\
\quad+\int_{0}^{t}\left\|p\left(N_{2}(\tau), \cdot\right)\left(n_{1}(\tau)-n_{2}(\tau)\right)\right\|_{\mathrm{TV}} \mathrm{d} \tau \\
+\int_{0}^{t}\left\|\int_{0}^{+\infty} \kappa(s, u)\left(p\left(N_{1}(\tau), \cdot\right)-p\left(N_{2}(\tau), \cdot\right)\right) n_{1}(\tau, \cdot) \mathrm{d} u\right\|_{\mathrm{TV}} \mathrm{d} \tau \\
\quad+\int_{0}^{t}\left\|\int_{0}^{+\infty} \kappa(s, u) p\left(N_{2}(\tau), \cdot\right)\left(n_{1}(\tau)-n_{2}(\tau)\right) \mathrm{d} u\right\|_{\mathrm{TV}} \mathrm{d} \tau,
\end{gathered}
$$

and with very similar arguments as before we obtain that

$$
\left\|n_{1}(t)-n_{2}(t)\right\|_{\mathrm{TV}} \leq\left\|n_{0}^{1}-n_{0}^{2}\right\|_{\mathrm{TV}}+4\|p\|_{\infty} \int_{0}^{t}\left\|n_{1}(\tau)-n_{2}(\tau)\right\|_{\mathrm{TV}} \mathrm{d} \tau
$$

Gronwall's inequality then implies (49).

\subsection{The linear equation}

The linear version of equation (3) obtained when $p=p(N, s)$ does not depend on $N$ :

$$
\left\{\begin{aligned}
\frac{\partial}{\partial t} n(t, s)+\frac{\partial}{\partial s} n(t, s) & +p(s) n(t, s)=\int_{0}^{+\infty} \kappa(s, u) p(u) n(t, u) \mathrm{d} u, \quad u, s, t>0 \\
n(t, s=0) & =0, \quad N=\int_{0}^{+\infty} p(s) n(t, s) \mathrm{d} s, \quad t>0 \\
n(t=0, s) & =n_{0}(s), \quad s \geq 0 .
\end{aligned}\right.
$$

\subsubsection{Well-posedness}

Similarly to Section 3.2.1, we can generalise slightly our concept of solution to include measures which are not necessarily nonnegative:

Definition 4.3. Assume that $p:[0,+\infty) \rightarrow[0,+\infty)$ is a bounded, nonnegative function satisfying (6) and $\kappa$ satisfies (9). A couple of functions $n \in \mathcal{C}\left([0, T), \mathcal{M}_{+}\left(\mathbb{R}_{0}^{+}\right)\right)$and 
$N \in \mathcal{C}([0, T),[0,+\infty))$, defined on an interval $[0, T)$ for some $T \in(0,+\infty]$, are called a mild measure solution to (3) with initial data $n_{0}(s) \in \mathcal{M}\left(\mathbb{R}_{0}^{+}\right)$if it satisfies $n(0)=n_{0}$

$$
n(t, s)=T_{t} n_{0}(s)+\int_{0}^{t} T_{t-\tau} A[n(\tau, \cdot)](s) \mathrm{d} \tau
$$

for all $t \in[0, T)$ where

$$
A[n](t, s):=-p(s) n(t, s)+\int_{0}^{+\infty} \kappa(s, u) p(u) n(t, u) \mathrm{d} u
$$

and

$$
N(t)=\int_{0}^{+\infty} p(s) n(t, s) \mathrm{d} s, \quad t \in[0, T) .
$$

By the existence result for (3) we have:

Theorem 4.4 (Well-posedness of (54) in measures). Assume that $p:[0,+\infty) \rightarrow[0,+\infty)$ is a bounded, nonnegative, Lipschitz function. Assume also that $\kappa$ satisfies (9). For any given initial data $n_{0} \in \mathcal{M}\left(\mathbb{R}^{+}\right)$there exists a unique measure solution $n \in \mathcal{C}\left([0,+\infty) ; \mathcal{M}\left(\mathbb{R}_{0}^{+}\right)\right)$ of the linear equation (54) in the sense of Definition 4.3. In addition, if $n$ is a mild measure solution to (54) defined on any interval $[0, T)$ then

$$
\|n(t)\|_{\mathrm{TV}} \leq\|n(0)\|_{\mathrm{TV}}, \quad \text { for all } t \in[0, T) .
$$

For the proof of this result one can follow the same procedure as in the proof of Theorem 3.11, so we omit it here. Theorem 4.4 allows us to define a $C_{0}$-semigroup $\left(S_{t}\right)_{t \geq 0}$ on $\mathcal{M}$, such that $S_{t}\left(n_{0}\right):=n(t)$ for any $n_{0} \in \mathcal{M}$ where $n(t)$ is the mild solution to (54) similarly as in Section 3.2.1.

Given $p$, we define $\mathcal{L}$ as the generator of the corresponding semigroup $S_{t}$, defined on its domain $\mathcal{D}(\mathcal{L})$. One can of course see that for sufficiently regular measures $n$,

$$
\mathcal{L} n(s)=\frac{\partial}{\partial s} n(s)+p(s) n(s)-\int_{0}^{+\infty} \kappa(s, u) p(u) n(u) \mathrm{d} u .
$$

Since the only unbounded operator involved in this expression is $\frac{\partial}{\partial s} n$, one sees that the domain $\mathcal{D}(\mathcal{L})$ can be described explicitly as

$$
\mathcal{D}(\mathcal{L}):=\left\{n \in \mathcal{M}\left(\mathbb{R}_{0}^{+}\right) \mid \frac{\partial}{\partial s} n \in \mathcal{M}\left(\mathbb{R}_{0}^{+}\right)\right\},
$$

where the derivative is taken in the sense of distributions on $\mathbb{R}$. Expression (57) is valid for all $n \in \mathcal{D}(\mathcal{L})$, again understanding the derivative in distributional sense.

Finally, for the arguments regarding the nonlinear equation (3) we will need a result on continuous dependence of the solutions of the linear equation (54) on the firing rate $p$ :

Theorem 4.5 (Continuous dependence with respect to $p$ for the linear equation). Let $p_{1}, p_{2}$ be bounded, nonnegative, Lipschitz functions. Assume also that $\kappa$ satisfies (9). For any given initial data $n_{0} \in \mathcal{M}\left(\mathbb{R}^{+}\right)$consider $n_{1}, n_{2}$ the two solutions to the linear equation (54) on $[0,+\infty)$ with firing rate $p_{1}, p_{2}$ respectively and initial data $n_{0}$. Assuming $\left\|p_{1}\right\|_{\infty} \neq 0$, it holds that

$$
\left\|n_{1}(t)-n_{2}(t)\right\|_{\mathrm{TV}} \leq \frac{\left\|n_{0}\right\|_{\mathrm{TV}}\left\|p_{1}-p_{2}\right\|_{\infty}}{\left\|p_{1}\right\|_{\infty}}\left(e^{2\left\|p_{1}\right\|_{\infty} t}-1\right) \quad \text { for all } t \geq 0 .
$$


Proof. With the obvious changes in notation, from (55) we have

$$
\begin{aligned}
\left\|n_{1}(t)-n_{2}(t)\right\|_{\mathrm{TV}} \leq \int_{0}^{t}\left\|T_{t-\tau} A_{1}\left[n_{1}(\tau, \cdot)\right]-T_{t-\tau} A_{2}\left[n_{2}(\tau, \cdot)\right]\right\|_{\mathrm{TV}} \mathrm{d} \tau & \\
& =\int_{0}^{t}\left\|A_{1}\left[n_{1}(\tau)\right]-A_{2}\left[n_{2}(\tau)\right]\right\|_{\mathrm{TV}} \mathrm{d} \tau .
\end{aligned}
$$

In a very similar way as the estimate we carried out for Theorem 4.2 , this last term can be estimated as

$$
\begin{aligned}
\| A_{1}\left[n_{1}(\tau)\right] & -A_{2}\left[n_{2}(\tau)\right] \|_{\mathrm{TV}} \\
& \leq 2\left\|p_{1}(s)\left(n_{1}(\tau, s)-n_{2}(\tau, s)\right)\right\|_{\mathrm{TV}}+2\left\|n_{2}(s)\left(p_{1}(s)-p_{2}(s)\right)\right\|_{\mathrm{TV}} \\
& \left.\quad \leq 2\left\|p_{1}\right\|_{\infty} \| n_{1}(\tau, \cdot)-n_{2}(\tau, \cdot)\right)\left\|_{\mathrm{TV}}+2\right\| n_{0}\left\|_{\mathrm{TV}}\right\| p_{1}-p_{2} \|_{\infty} .
\end{aligned}
$$

Hence, calling $m(t) \equiv m(t):=\left\|n_{1}(t, \cdot)-n_{2}(t, \cdot)\right\|_{\mathrm{TV}}$ and $K:=\left\|n_{0}\right\|_{\mathrm{TV}}\left\|p_{1}-p_{2}\right\|_{\infty}$, we have

$$
m(t) \leq 2\left\|p_{1}\right\|_{\infty} \int_{0}^{t} m(\tau) \mathrm{d} \tau+2 t K
$$

Gronwall's Lemma then shows that

$$
m(t) \leq \frac{K}{\left\|p_{1}\right\|_{\infty}}\left(e^{2\left\|p_{1}\right\|_{\infty} t}-1\right) .
$$

\section{Stationary solutions for the linear equation}

Definition 4.6. A stationary solution to $(54) n_{*} \in \mathcal{M}$ is defined as such that $n_{*} \in \mathcal{D}(\mathcal{L})$ and

$$
\mathcal{L} n_{*}=0 .
$$

We remark that Proposition 4.8 below implies that the linear equation (54) has a unique stationary solution in the space of probabilities on $[0,+\infty)$ (for $p$ bounded, Lipschitz, satisfying (8) and $\kappa$ satisfying (9)); $n_{*}$ is the only stationary solution up to a constant factor within the set of all finite measures.

\subsubsection{Positive lower bound}

Analogous to Section 3.2, we want to show that for a given positive initial distribution, solutions of (54) after some time have a positive lower bound, so that the semigroup $S_{t}\left(n_{0}\right)$ satisfies the Doeblin's condition given in (12).

Lemma 4.7. Let $p:[0,+\infty) \longrightarrow[0,+\infty)$ be a bounded, Lipschitz function satisfying (7) and (8). We assume also that $\kappa$ satisfies (9) and (10). Consider the semigroup defined as $S_{t}\left(n_{0}\right):=n(t)$ for any $n_{0} \in \mathcal{M}$. Then $S_{t_{0}}$ satisfy the Doeblin condition (12) for $t_{0}=2 s_{*}$ and $\alpha=\epsilon \delta p_{\min }\left(s_{*}-\delta\right) e^{-p_{\max } t_{0}}$. More precisely, for $t_{0}=2 s_{*}$ we have

$$
S_{2 s_{*}} n_{0}(s) \geq \epsilon \delta p_{\min } e^{-2 p_{\max } s_{*}} \mathbb{1}_{\left\{\delta<s<s_{*}\right\}}
$$

for all probability measures $n_{0}$ on $[0,+\infty)$. 
Proof. Since for $s, t>0$, it holds true for solutions of (54) that

$$
\frac{\partial}{\partial t} n(t, s)+\frac{\partial}{\partial s} n(t, s) \geq-p(s) n(t, s) .
$$

Moreover, solutions of $(54)$ satisfy $n(t, s) \geq \tilde{n}(t, s)$ where the equation on $\tilde{n}(t, s)$ was defined in (36) of Lemma 3.12. By the same argument we have for $t>s_{*}, N(t) \geq$ $p_{\min } e^{-p_{\max } t}$.

We consider the same semigroup $\tilde{S}_{t}$ associated to (36). Then, solutions of (54) satisfy

$$
\begin{aligned}
n(t, s)=\tilde{S}_{t} n_{0}(s)+\int_{0}^{t} \tilde{S}_{t-\tau}\left(\int_{0}^{+\infty} \kappa(., u) p(u)\right. & n(t, u) \mathrm{d} u)(s) \mathrm{d} \tau \\
& \geq \tilde{S}_{t} n_{0}(s)+\int_{0}^{t} \tilde{S}_{t-\tau}\left(\epsilon N(\tau) \mathbb{1}_{\{s \leq \delta\}}\right) \mathrm{d} \tau
\end{aligned}
$$

since

$$
\begin{array}{r}
\int_{0}^{+\infty} \kappa(s, u) p(u) n(t, u) \mathrm{d} u \geq \int_{0}^{+\infty} \phi(s) p(u) n(t, u) \mathrm{d} u \geq \epsilon \mathbb{1}_{\{s \leq \delta\}} \int_{0}^{+\infty} p(u) n(t, u) \mathrm{d} u \\
=\epsilon \mathbb{1}_{\{s \leq \delta\}} N(t) .
\end{array}
$$

Then for $t>s+s_{*}$ and $s>\delta$ we have

$$
\begin{array}{r}
n(t, s) \geq \int_{0}^{t} \tilde{S}_{t-\tau}\left(\epsilon N(\tau) \mathbb{1}_{\{s \leq \delta\}}\right) \mathrm{d} \tau \geq \int_{s_{*}}^{t} \tilde{S}_{t-\tau}\left(\epsilon \mathbb{1}_{\{s \leq \delta\}} p_{\min } e^{-p_{\max } \tau}\right) \mathrm{d} \tau \\
\geq \epsilon p_{\min } \int_{s_{*}}^{t} e^{-p_{\max } \tau} e^{-p_{\max }(t-\tau)} \mathbb{1}_{\{0<s-t+\tau \leq \delta\}} \mathrm{d} \tau=\epsilon p_{\min } e^{-p_{\max } t} \int_{s_{*}}^{t} \mathbb{1}_{\{0<s-t+\tau \leq \delta\}} \mathrm{d} \tau \\
\quad=\epsilon \delta p_{\min } e^{-p_{\max } t_{1}} \mathbb{1}_{\left\{\delta<s<t_{0}-s_{*}\right\}} .
\end{array}
$$

Hence for $t=2 s_{*}$ and $\delta<s<s_{*}$ we obtain the result.

\subsubsection{Spectral gap}

We again obtain a spectral gap property as a consequence of Theorem 2.3:

Proposition 4.8. Let $n_{0} \in \mathcal{M}\left(\mathbb{R}^{+}\right)$be the initial data given for (54). We assume that $p$ is a nonnegative, Lipschitz function satisfying (6)-(8) and $\kappa$ satisfies (9), (10). Then, there exists a unique probability measure $n_{*} \in \mathcal{P}([0,+\infty))$ which is a stationary solution to (54), and any other stationary solution is a multiple of it. Also for

$$
C=\frac{1}{1-\alpha}>1, \text { and } \lambda=-\frac{\log (1-\alpha)}{t_{0}},
$$

we have

$$
\left\|S_{t}\left(n_{0}-n_{*}\right)\right\|_{T V} \leq C e^{-\lambda t}\left\|n_{0}-n_{*}\right\|_{\mathrm{TV}}, \text { for all } t \geq 0 .
$$

In addition, for $t_{0}:=2 s_{*}$ we have

$$
\left\|S_{t_{0}}\left(n_{1}-n_{2}\right)\right\|_{\mathrm{TV}} \leq(1-\alpha)\left\|n_{1}-n_{2}\right\|_{\mathrm{TV}}
$$

for any probability distributions $n_{1}, n_{2}$, and with

$$
\alpha:=\epsilon \delta p_{\min }\left(s_{*}-\delta\right) e^{-2 p_{\max } s_{*}} .
$$

Proof. Lemma 4.7 ensures the operator $S_{t_{0}}$ satisfies the Doeblin condition (12) for $t_{0}=$ $2 s_{*}$. We obtain the result by applying Theorem 2.3 . 


\subsection{Stationary solutions for the nonlinear equation}

Definition 4.9. We say that a pair $\left(n_{*}, N_{*}\right)$, where $n_{*} \in \mathcal{M}_{+}\left(\mathbb{R}_{0}^{+}\right)$and $N_{*} \geq 0$, is a stationary solution to $(3)$ if $n_{*} \in \mathcal{D}(\mathcal{L})$ and

$$
\mathcal{L}_{N_{*}} n_{*}=0, \quad N_{*}=\int_{0}^{+\infty} p\left(N_{*}, s\right) n_{*}(s) \mathrm{d} s,
$$

where $\mathcal{L}_{N_{*}}$ is the semigroup generator associated to $p(s) \equiv p\left(N_{*}, s\right)$ (see Theorem 4.4 and the following remarks; observe that the domain $\mathcal{D}(\mathcal{L})$ does not depend on the value of $N_{*}$ ). We say that $N_{*}$ is the global neural activity associated to the stationary solution.

We give the following theorem for existence and uniqueness of stationary solutions:

Theorem 4.10. Assume (5), (6), (8), (9), and also that

$$
L<\left(1+\frac{C p_{\max }}{\alpha p_{\min }}\right)^{-1}
$$

where $C:=e^{4 p_{\max } s_{*}}$ and $\alpha$ is given by Proposition 4.8. Then there exists a unique stationary solution $\left(n_{*}, N_{*}\right)$ of (3) such that $n_{*}$ is a probability measure.

Proof. Proposition 4.8 ensures that for a fixed $N_{*}$, there exists a unique probability stationary solution of the corresponding linear problem. We prove the existence of a stationary solution by recovering $N_{*}$ from $n_{*}$ and carrying out a fixed-point argument. We define a map $\Upsilon:[0,+\infty) \longrightarrow[0,+\infty)$, by

$$
\Upsilon(N):=\int_{0}^{+\infty} p(N, s) n(s) \mathrm{d} s
$$

where $n$ is the unique probability measure which is an equilibrium of the linear problem associated to $p(s) \equiv p(N, s)$. We notice that the statement we wish to prove is equivalent to the fact that $\Upsilon$ has a unique fixed point.

Let us show that this map is contractive. For any $N_{1}, N_{2} \geq 0$,

$$
\begin{aligned}
& \left|\Upsilon\left(N_{1}\right)-\Upsilon\left(N_{2}\right)\right|=\left|\int_{0}^{+\infty}\left(p\left(N_{1}, s\right) n_{1}(s)-p\left(N_{2}, s\right) n_{2}(s)\right) \mathrm{d} s\right| \\
& \leq \int_{0}^{+\infty}\left|\left(p\left(N_{1}, s\right)-p\left(N_{2}, s\right)\right) n_{2}(s)\right| \mathrm{d} s+\int_{0}^{+\infty}\left|p\left(N_{1}, s\right)\left(n_{1}(s)-n_{2}(s)\right)\right| \mathrm{d} s \\
& \leq L\left|N_{1}-N_{2}\right|+p_{\max }|| n_{1}-n_{2} \|_{\mathrm{TV}} .
\end{aligned}
$$

Now, we will prove later that

$$
\left\|n_{1}-n_{2}\right\|_{\mathrm{TV}} \leq \frac{L C}{\alpha p_{\min }}\left|N_{1}-N_{2}\right|
$$

where $C:=e^{4 p_{\max } s_{*}}$ and $\alpha$ is the one from Proposition 4.8. This implies that

$$
\left|\Upsilon\left(N_{1}\right)-\Upsilon\left(N_{2}\right)\right| \leq L\left(1+\frac{C p_{\max }}{\alpha p_{\min }}\right)\left|N_{1}-N_{2}\right|
$$


which makes $\Upsilon$ a contraction operator if $L$ satisfies the inequality in the statement. So in order to complete the proof we only need to show (60). For this we define the two operators

$$
P_{1}(n):=S_{t_{0}}^{1} n-n, \quad P_{2}(n):=S_{t_{0}}^{2} n-n,
$$

where for $i=1,2,\left(S_{t}^{i}\right)_{t \geq 0}$ is the linear semigroup given by Theorem 4.4, associated to the firing rate $p_{i}(s):=p\left(N_{i}, s\right)$, and $t_{0}:=2 s_{*}$ is the time mentioned in Proposition 4.8. We use that, since $n_{1}, n_{2}$ are equilibria for the linear equations with $p_{1}, p_{2}$,

$$
0=P_{1}\left(n_{1}\right)=P_{2}\left(n_{2}\right)
$$

so that

$$
\begin{aligned}
0=\left\|P_{1}\left(n_{1}\right)-P_{2}\left(n_{2}\right)\right\|_{\mathrm{TV}}=\| P_{1}\left(n_{1}-n_{2}\right)+ & \left(P_{1}-P_{2}\right)\left(n_{2}\right) \|_{\mathrm{TV}} \\
& \geq\left\|P_{1}\left(n_{1}-n_{2}\right)\right\|_{\mathrm{TV}}-\left\|\left(P_{1}-P_{2}\right) n_{2}\right\|_{\mathrm{TV}},
\end{aligned}
$$

which implies

$$
\left\|P_{1}\left(n_{1}-n_{2}\right)\right\|_{\mathrm{TV}} \leq\left\|\left(P_{1}-P_{2}\right) n_{2}\right\|_{\mathrm{TV}} .
$$

Then by Proposition 4.8 we have

$$
\left\|P_{1}\left(n_{1}-n_{2}\right)\right\|_{\mathrm{TV}} \geq\left\|n_{1}-n_{2}\right\|_{\mathrm{TV}}-\left\|S_{t_{0}}^{1}\left(n_{1}-n_{2}\right)\right\|_{\mathrm{TV}} \geq \alpha\left\|n_{1}-n_{2}\right\|_{\mathrm{TV}},
$$

since $\int n_{1} \mathrm{~d} s=\int n_{2} \mathrm{~d} s=1$, where $\alpha$ is the one in Proposition 4.8. On the other hand, by $(58)$,

$$
\begin{array}{r}
\left\|\left(P_{1}-P_{2}\right) n_{2}\right\|_{\mathrm{TV}}=\left\|\left(S_{t_{0}}^{1}-S_{t_{0}}^{2}\right) n_{2}\right\|_{\mathrm{TV}} \leq \frac{\left\|n_{2}\right\|_{\mathrm{TV}}\left\|p_{1}-p_{2}\right\|_{\infty}}{\left\|p_{1}\right\|_{\infty}}\left(e^{2\left\|p_{1}\right\|_{\infty} t_{0}}-1\right) \\
\leq \frac{L\left|N_{1}-N_{2}\right|}{p_{\min }} e^{2 p_{\max } t_{0}} .
\end{array}
$$

Using the last two equations in (61),

$$
\left\|n_{1}-n_{2}\right\|_{\mathrm{TV}} \leq \frac{1}{\alpha}\left\|\left(P_{1}-P_{2}\right) n_{2}\right\|_{\mathrm{TV}} \leq \frac{L\left|N_{1}-N_{2}\right|}{\alpha p_{\min }} e^{2 p_{\max } t_{0}},
$$

which proves (60). Therefore $\Upsilon$ has a unique fixed point, and hence (3) has a unique stationary solution.

\subsection{Asymptotic behaviour}

In this section we prove Theorem 1.1 for equation (3). We define two operators in the following way:

$$
\begin{aligned}
\mathcal{L}_{N(t)} n(t, s) & :=\partial_{t} n(t, s)=-\partial_{s} n(t, s)-p(N(t), s) n(t, s)+\int \kappa(s, u) p(N(t), u) n(t, u) d u \\
\mathcal{L}_{N_{*}} \bar{n}(s) & :=-\partial_{s} \bar{n}(s)-p\left(N_{*}, s\right) \bar{n}(s)+\int \kappa(s, u) p\left(N_{*}, u\right) \bar{n}(s) d u
\end{aligned}
$$

We rewrite (3) as

$$
\frac{\partial}{\partial t} n(t, s)=\mathcal{L}_{N(t)} n(t, s)=\mathcal{L}_{N_{*}} n(t, s)-\left(\mathcal{L}_{N_{*}}-\mathcal{L}_{N(t)}\right) n(t, s) .
$$


Then, similarly as in Section 3.4 by Ball (1977) we may use Duhamel's formula and write the solution as

$$
n(t, s)=S_{t} n_{0}(s)+\int_{0}^{t} S_{t-\tau} h(\tau, s) \mathrm{d} \tau
$$

where $S_{t} n_{0}(s):=e^{\mathcal{L}_{N_{*}}} n_{0}(s)$ and $\bar{n}$ is the solution to linear problem, $\mathcal{L}_{N_{*}}$ is acting on $n(t, s)$. Also,

$$
\begin{aligned}
& h(t, s):=\left(\mathcal{L}_{N_{*}}-\mathcal{L}_{N(t)}\right) n(t, s) \\
& =\left(p(N(t), s)-p\left(N_{*}, s\right)\right) n(t, s)+\int_{0}^{+\infty} \kappa(s, u)\left(p\left(N_{*}, u\right)-p(N(t), u)\right) n(t, u) \mathrm{d} u .
\end{aligned}
$$

Then we give the following lemma:

Lemma 4.11. Assume that (6) and (8) hold true for a Lipschitz function $p$ and $\kappa$ satisfies (9). Then $h$, which is defined by (64), satisfies

$$
\|h(t)\|_{\mathrm{TV}} \leq \tilde{C}\left\|n(t)-n_{*}\right\|_{\mathrm{TV}}
$$

where $\tilde{C}=2 p_{\max } \frac{L}{1-L}$. Moreover $\int_{0}^{+\infty} h(t, s) \mathrm{d} s=0$.

Proof.

$$
\begin{aligned}
& \|h(t)\|_{\mathrm{TV}}=\left\|\left(\mathcal{L}_{N_{*}}-\mathcal{L}_{N(t)}\right) n(t, s)\right\|_{\mathrm{TV}} \\
& \leq\left\|\left(p(N(t), s)-p\left(N_{*}, s\right)\right) n(t, s)\right\|_{\mathrm{TV}}+\left\|\int_{0}^{+\infty} \kappa(s, u)\left(p\left(N_{*}, u\right)-p(N(t), u)\right) n(t, u) d u\right\|_{T V} \\
& \quad \leq L\|n(t)\|_{\mathrm{TV}}\left|N_{*}-N(t)\right|+L\|n(t)\|_{\mathrm{TV}}\left|N_{*}-N(t)\right| \\
& \quad \leq 2 p_{\max } \frac{L\|n(t)\|_{\mathrm{TV}}}{1-L\|n(t)\|_{\mathrm{TV}}}\left\|n(t)-n_{*}\right\|_{\mathrm{TV}}=2 p_{\max } \frac{L}{1-L}\left\|n(t)-n_{*}\right\|_{\mathrm{TV}}
\end{aligned}
$$

Since

$$
\begin{array}{r}
\left|N_{*}-N(t)\right|=\left|\int_{0}^{+\infty} p\left(N_{*}, s\right) n_{*}(s) d s-\int_{0}^{+\infty} p(N(t), s) n(t, s) d s\right| \\
\leq\left|\int_{0}^{+\infty}\left(p\left(N_{*}, s\right) n_{*}(s)+\left(p\left(N_{*}, s\right) n(t, s)-p\left(N_{*}, s\right) n(t, s)\right) p(N(t), s) n(t, s)\right) d s\right| \\
\leq\left|\int_{0}^{+\infty} p\left(N_{*}, s\right)\left(n_{*}(s)-n(t, s)\right) d s\right|+\mid \int_{0}^{+\infty}\left(p\left(N_{*}, s\right)-p(N(t), s) n(t, s) d s \mid\right. \\
\leq p_{\max }\left\|n(t)-n_{*}\right\|_{\mathrm{TV}}+L\left|N_{*}-N(t)\right|\|n\|_{\mathrm{TV}}
\end{array}
$$

implies that

$$
\left|N_{*}-N(t)\right| \leq \frac{p_{\max }}{1-L\|n(t)\|_{T V}}\left\|n(t)-n_{*}\right\|_{\mathrm{TV}}=\frac{p_{\max }}{1-L}\left\|n(t)-n_{*}\right\|_{\mathrm{TV}}
$$


since $\|n(t)\|_{\mathrm{TV}}=\left\|n_{*}\right\|_{\mathrm{TV}}=1$. Moreover we have

$$
\begin{gathered}
\int_{0}^{+\infty} h(t, s) \mathrm{d} s=\int_{0}^{+\infty} p(N(t), s) \bar{n}(t, s) \mathrm{d} s-\int_{0}^{+\infty} p\left(N_{*}, s\right) \bar{n}(t, s) \mathrm{d} s \\
+\int_{0}^{+\infty} \int_{0}^{+\infty} \kappa(s, u) p\left(N_{*}, u\right) \bar{n}(t, u) \mathrm{d} u \mathrm{~d} s-\int_{0}^{+\infty} \int_{0}^{+\infty} \kappa(s, u) p(N(t), u) \bar{n}(t, u) \mathrm{d} u \mathrm{~d} s \\
=N(t)-\int_{0}^{+\infty} p\left(N_{*}, s\right) \bar{n}(t, s) \mathrm{d} s \\
+\int_{0}^{+\infty}\left(\int_{0}^{u} \kappa(s, u) \mathrm{d} s\right) p\left(N_{*}, u\right) \bar{n}(t, u) \mathrm{d} u+\int_{0}^{+\infty} \int_{0}^{u} \kappa(s, u) \mathrm{d} s p(N(t), u) \bar{n}(t, u) \mathrm{d} u \\
=N(t)-\int_{0}^{+\infty} p\left(N_{*}, s\right) \bar{n}(t, s) \mathrm{d} s+\int_{0}^{+\infty} p\left(N_{*}, u\right) \bar{n}(t, u) \mathrm{d} u-N(t)=0 .
\end{gathered}
$$

Proof of Theorem 1.1 for (3). We subtract the unique probability stationary solution from both sides of (63):

$$
n(t, s)-n_{*}(s)=S_{t} n_{0}(s)-n_{*}(s)+\int_{0}^{t} S_{t-\tau} h(\tau, s) \mathrm{d} \tau .
$$

We take the total variation norm and obtain

$$
\left\|n(t)-n_{*}\right\|_{\mathrm{TV}} \leq\left\|S_{t} n_{0}-n_{*}\right\|_{\mathrm{TV}}+\left\|\int_{0}^{t} S_{t-\tau} h(\tau, s) \mathrm{d} \tau\right\|_{\mathrm{TV}}
$$

Then by Proposition 4.8 and Lemma 4.11 we have

$$
\begin{aligned}
&\left\|n(t)-n_{*}\right\|_{\mathrm{TV}} \leq C e^{-\lambda t}\left\|n_{0}-n_{*}\right\|_{\mathrm{TV}}+\int_{0}^{t}\left\|S_{t-\tau} h(\tau, s)\right\|_{\mathrm{TV}} \mathrm{d} \tau \\
& \leq C e^{-\lambda t}\left\|n_{0}-n_{*}\right\|_{\mathrm{TV}}+\int_{0}^{t} e^{-\lambda(t-\tau)}\|h(\tau, s)\|_{\mathrm{TV}} \mathrm{d} \tau \\
& \leq C e^{-\lambda t}\left\|n_{0}-n_{*}\right\|_{\mathrm{TV}}+\tilde{C} \int_{0}^{t} e^{-\lambda(t-\tau)}\left\|n(\tau)-n_{*}\right\|_{\mathrm{TV}} \mathrm{d} \tau
\end{aligned}
$$

Therefore, by Gronwall's inequality

$$
\left\|n(t)-n_{*}\right\|_{\mathrm{TV}} \leq C e^{-(\lambda-\tilde{C}) t}\left\|n_{0}-n_{*}\right\|_{\mathrm{TV}}
$$

\section{Acknowledgements}

The authors would like to thank Susana Gutiérrez and Thibault Bourgeron for several useful discussions on the models studied in this paper. JAC and HY were supported by projects MTM2014-52056-P and MTM2017-85067-P, funded by the Spanish government and the European Regional Development Fund. HY was also supported by the Basque Government through the BERC 2014-2017 program and by Spanish Ministry of Economy and Competitiveness MINECO: BCAM Severo Ochoa excellence accreditation SEV-2013-0323 and by "la Caixa Grant". 


\section{References}

J. M. Ball. Strongly continuous semigroups, weak solutions, and the variation of constants formula. Proceedings of the American Mathematical Society, 63(2):370-373, 1977.

V. Bansaye, B. Cloez, and P. Gabriel. Ergodic behavior of non-conservative semigroups via generalized doeblin's conditions. November 2017, arXiv:1710.05584v3.

A. Brännström, L. Carlsson, and D. Simpson. On the convergence of the escalator boxcar train. SIAM Journal on Numerical Analysis, 51(6):3213-3231, 2013.

R. Brette and W. Gerstner. Adaptive exponential integrate-and-fire model as an effective description of neuronal activity. Journal of Neurophysiology, 94(5):3637-3642, 2005. ISSN 0022-3077.

N. Brunel. Dynamics of sparsely connected networks of excitatory and inhibitory spiking neurons. Journal of Computational Neuroscience, 8(3):183-208, May 2000. ISSN 1573-6873.

N. Brunel and V. Hakim. Fast global oscillations in networks of integrate-and-fire neurons with low firing rates. Neural Computation, 11(7):1621-1671, 1999.

J. A. Cañizo, J. A. Carrillo, and S. Cuadrado. Measure solutions for some models in population dynamics. Acta Applicandae Mathematicae, 123(1):141-156, February 2013. ISSN 0167-8019, arXiv:1112.0522.

M. J. Cáceres and R. Schneider. Blow-up, steady states and long time behaviour of excitatory-inhibitory nonlinear neuron models. Kinetic \&6 Related Models, 10(3):587612, 2017. ISSN 1937-5093.

M. J. Cáceres and R. Schneider. Analysis and a numerical solver for excitatoryinhibitory networks with delay and refractory periods. ESAIM: M2AN, 2018.

M. J. Cáceres, J. A. Carrillo, and B. Perthame. Analysis of nonlinear noisy integrate\&fire neuron models: blow-up and steady states. The Journal of Mathematical Neuroscience, 1(1):7, 2011. ISSN 2190-8567.

V. Calvez, N. Lenuzza, M. Doumic, J. P. Deslys, F. Mouthon, and B. Perthame. Prion dynamics with size dependency-strain phenomena. Journal of Biological Dynamics, 4(1):28-42, 2010.

J. A. Carrillo, M. González, M. P. Gualdani, and M. E. Schonbek. Classical solutions for a nonlinear fokker-planck equation arising in computational neuroscience. Communications in Partial Differential Equations, 38(3):385-409, March 2013.

J. A. Carrillo, P. Gwiazda, and A. Ulikowska. Splitting-particle methods for structured population models: Convergence and applications. Mathematical Models and Methods in Applied Sciences, 24(11):2171-2197, 2014.

J. Chevallier. Mean-field limit of generalized hawkes processes. Stochastic Processes and their Applications, 127(12):3870 - 3912, 2017. ISSN 0304-4149. 
J. Chevallier, M. J. Cáceres, M. Doumic, and P. Reynaud-Bouret. Microscopic approach of a time elapsed neural model. Mathematical Models and Methods in Applied Sciences, 25(14):2669-2719, 2015.

O. Diekmann and M. Gyllenberg. The second half-with a quarter of a century delay. Math. Model. Nat. Phenom., 3(7):36-48, 2008.

M. Doumic-Jauffret and P. Gabriel. Eigenelements of a general aggregationfragmentation model. Mathematical Models and Methods in Applied Sciences, 20 (5):757-783, May 2010.

H. Engler, J. Prüss, and G. F. Webb. Analysis of a model for the dynamics of prions ii. Journal of Mathematical Analysis and Applications, 324(1):98-117, December 2006. ISSN 0022-247X.

J. Z. Farkas and T. Hagen. Stability and regularity results for a size-structured population model. Journal of Mathematical Analysis and Applications, 328(1):119-136, 2007. ISSN 0022-247X.

P. Gabriel. Long-time asymptotics for nonlinear growth-fragmentation equations. Communications in Mathematical Sciences, 10(3):787-820, 2012.

P. Gabriel. Measure solutions to the conservative renewal equation. ESAIM: ProcS, 62: 68-78, 2018.

P. Gwiazda and E. Wiedemann. Generalized entropy method for the renewal equation with measure data. Communications in Mathematical Sciences, 15(2):577-586, April 2016.

P. Gwiazda, J. Jabłoński, A. Marciniak-Czochra, and A. Ulikowska. Analysis of particle methods for structured population models with nonlocal boundary term in the framework of bounded lipschitz distance. Numerical Methods for Partial Differential Equations, 30(6):1797-1820, 2014. ISSN 1098-2426.

M. Gyllenberg and G. F. Webb. A nonlinear structured population model of tumor growth with quiescence. Journal of Mathematical Biology, 28(6):671-694, Sep 1990. ISSN 1432-1416.

M. Hairer and J. C. Mattingly. Yet another look at harris' ergodic theorem for markov chains. In Seminar on Stochastic Analysis, Random Fields and Applications VI, pages 109-117, Basel, 2011. Springer Basel. ISBN 978-3-0348-0021-1.

T. E. Harris. The existence of stationary measures for certain markov processes. In Proceedings of the Third Berkeley Symposium on Mathematical Statistics and Probability, Volume 2: Contributions to Probability Theory, pages 113-124, Berkeley, Calif., 1956. University of California Press.

M. Iannelli. Mathematical theory of age-structured population dynamics. Applied mathematics monographs. Giardini editori e stampatori; Pisa, 1995. ISBN 9788842702504.

P. Laurençot and C. Walker. Well-posedness for a model of prion proliferation dynamics. Journal of Evolution Equations, 7(2):241-264, May 2007. 
T. Lorenz. Mutational analysis. A joint framework for Cauchy problems in and beyond vector spaces, volume 1996. Springer-Verlag Berlin; Heidelberg, January 2010. ISBN 978-3-642-12471-6.

J. A. J. Metz and O. Diekmann. The Dynamics of physiologically structured populations. Springer-Verlag Berlin; New York, 1986. ISBN 35401678620387167862.

P. Michel. Existence of a solution to the cell division eigenproblem. Mathematical Models and Methods in Applied Sciences, 16(supp01):1125-1153, 2006.

S. Mischler and Q. Weng. Relaxation in time elapsed neuron network models in the weak connectivity regime. Acta Applicandae Mathematicae, 157(1):45-74, October 2018. ISSN 1572-9036.

K. Pakdaman, B. Perthame, and D. Salort. Dynamics of a structured neuron population. Nonlinearity, 23(1):55-75, January 2010. ISSN 0951-7715.

K. Pakdaman, B. Perthame, and D. Salort. Relaxation and self-sustained oscillations in the time elapsed neuron network model. SIAM Journal on Applied Mathematics, 73(3):1260-1279, June 2013. ISSN 0036-1399.

K. Pakdaman, B. Perthame, and D. Salort. Adaptation and fatigue model for neuron networks and large time asymptotics in a nonlinear fragmentation equation. The Journal of Mathematical Neuroscience, 4(1):1-26, 2014.

B. Perthame. Transport Equations in Biology. Frontiers in Mathematics. Birkhäuser Basel, 1 edition, 2006.

B. Perthame and L. Ryzhik. Exponential decay for the fragmentation or cell-division equation. Journal of Differential Equations, 210(1):155-177, 2005. ISSN 0022-0396.

J. Pham, K. Pakdaman, J. Champagnat, and J. F. Vibert. Activity in sparsely connected excitatory neural networks: effect of connectivity. Neural Networks, 11(3): 415-434, 1998. ISSN 0893-6080.

C. Rossant, D. F. Goodman, B. Fontaine, J. Platkiewicz, A. Magnusson, and R. Brette. Fitting neuron models to spike trains. Frontiers in Neuroscience, 5:9, 2011. ISSN 1662-453X.

G. Simonett and C. Walker. On the solvability of a mathematical model for prion proliferation. Journal of Mathematical Analysis and Applications, 324(1):580-603, 2006. ISSN 0022-247X.

D. W. Stroock. An Introduction to Markov Processes. Springer-Verlag Berlin; Heidelberg, 2005. ISBN 9783540234517.

H. R. Thieme. Mathematics in Population Biology. Mathematical Biology Series. Princeton University Press, 2003. ISBN 9780691092911.

H. C. Tuckwell. Introduction to Theoretical Neurobiology: Volume 2, Nonlinear and Stochastic Theories. Cambridge Studies in Mathematical Biology. Cambridge University Press, 1 edition, 1988. ISBN 9780521352178. 
G. F. Webb. Theory of nonlinear age-dependent population dynamics. M. Dekker New York, 1985. ISBN 978-0824772901.

Q Weng. General time elapsed neuron network model: well-posedness and strong connectivity regime. ArXiv e-prints, December 2015, arXiv:1512.07112v3.

José A. Cañizo. Departamento de Matemática Aplicada, Universidad de Granada, 18071 Granada, Spain. Email address: canizo@ugr.es

Havva Yoldaş. Basque Center for Applied Mathematics, Alameda de Mazarredo 14, 48009 Bilbao, Spain \& Departamento de Matemática Aplicada, Universidad de Granada, 18071 Granada, Spain. Email address: hyoldas@bcamath.org 